\title{
Radio continuum sources behind the Large Magellanic Cloud
}

\author{
M. D. Filipović ${ }^{(0)},{ }^{1 \star}$ I. S. Bojičić ${ }^{\circledR},{ }^{1}$ K. R. Grieve, ${ }^{1}$ R. P. Norris ${ }^{\circledR}, 1,2$ N. F. H. Tothill, ${ }^{1}$ D. Shobhana, ${ }^{1}$ \\ L. Rudnick ${ }^{\oplus}, 3$ I. Prandoni ${ }^{\oplus},{ }^{4}$ H. Andernach ${ }^{\circledR},{ }^{5}$ N. Hurley-Walker, ${ }^{6}$ R. Z. E. Alsaberi ${ }^{\circledR},{ }^{1}$ C. \\ S. Anderson ${ }^{\circledR},{ }^{2}$ J. D. Collier ${ }^{\circledR},{ }^{1,7}$ E. J. Crawford ${ }^{\circledR},{ }_{1}^{1}$ B.-Q. For ${ }^{\circledR},{ }^{8,9}$ T. J. Galvin ${ }^{\circledR}, 6$ F. Haberl, ${ }^{10}$ A. \\ M. Hopkins, ${ }^{11,1}$ A. Ingallinera ${ }^{\circledR},{ }^{12}$ P. J. Kavanagh, ${ }^{13}$ B. S. Koribalski ${ }^{\circledR},{ }^{1,2}$ R. Kothes, ${ }^{14}$ D. Leahy ${ }^{\circledR},{ }^{15}$ \\ H. Leverenz ${ }^{\circledR},{ }^{1}$ P. Maggi, ${ }^{16}$ C. Maitra ${ }^{\circledR},{ }^{10}$ J. Marvil, ${ }^{17}$ T. G. Pannuti, ${ }^{18}$ L. A. F. Park, ${ }^{1}$ J. L. Payne, ${ }^{1}$ \\ C. M. Pennock ${ }^{\circledR},{ }^{19}$ S. Riggi ${ }^{\circledR},{ }^{12}$ G. Rowell, ${ }^{20}$ H. Sano ${ }^{\circledR},{ }^{21}$ M. Sasaki ${ }^{\circledR}, 22$ L. Staveley-Smith ${ }^{\circledR}, 8^{7,8}$ \\ C. Trigilio, ${ }^{12}$ G. Umana, ${ }^{12}$ D. Urošević, ${ }^{23,24}$ J. Th. van Loon ${ }^{19}$ and E. Vardoulaki ${ }^{25}$
}

Affiliations are listed at the end of the paper

Accepted 2021 July 22. Received 2021 July 22; in original form 2021 March 18

\begin{abstract}
We present a comprehensive multifrequency catalogue of radio sources behind the Large Magellanic Cloud (LMC) between 0.2 and $20 \mathrm{GHz}$, gathered from a combination of new and legacy radio continuum surveys. This catalogue covers an area of $\sim 144 \mathrm{deg}^{2}$ at angular resolutions from 45 arcsec to $\sim 3$ arcmin. We find 6434 discrete radio sources in total, of which 3789 are detected at two or more radio frequencies. We estimate the median spectral index ( $\alpha$; where $S_{v} \sim \nu^{\alpha}$ ) of $\alpha=-0.89$ and mean of $-0.88 \pm 0.48$ for 3636 sources detected exclusively at two frequencies $(0.843$ and $1.384 \mathrm{GHz})$ with similar resolution [full width at half-maximum (FWHM) 40-45 arcsec]. The large frequency range of the surveys makes it an effective tool to investigate Gigahertz Peak Spectrum (GPS), Compact Steep Spectrum (CSS), and Infrared Faint Radio Source (IFRS) populations within our sample. We find 10 GPS candidates with peak frequencies near $5 \mathrm{GHz}$, from which we estimate their linear size. 1866 sources from our catalogue are CSS candidates with $\alpha<-0.8$. We found six candidates for High Frequency Peaker (HFP) sources, whose radio fluxes peak above $5 \mathrm{GHz}$ and no sources with unconstrained peaks and $\alpha>0.5$. We found optical counterparts for 343 of the radio continuum sources, of which 128 have a redshift measurement. Finally, we investigate the population of 123 IFRSs found in this study.
\end{abstract}

Key words: catalogues - galaxies: active-Magellanic Clouds - radio continuum: general.

\section{INTRODUCTION}

Various peoples of the Southern hemisphere, including Australian Aborigines, incorporated the Magellanic Clouds (MCs) as an important part of their culture. These obvious astronomical objects of the southern sky were seen by Portuguese explorer Ferdinand Magellan in the $16^{\text {th }}$ century. He described the MCs as the most vivid celestial objects of the southern sky seen during his mesmerizing expeditions (for more details on historical observations of MCs, see Filipovic et al. 1996a).

The Large Magellanic Cloud (LMC) is thought to be a barred spiral galaxy, dynamically disrupted due to its proximity to the Milky Way (MW) and other interactions with the Small Magellanic Cloud (SMC; Mathewson, Cleary \& Murray 1974). The LMC is currently $\sim 50 \mathrm{kpc}$ (Pietrzyński et al. 2013, 2019; Riess et al. 2019) from the centre of the MW, while the SMC is further away at a distance of $\sim 60 \mathrm{kpc}$ (Hilditch, Howarth \& Harries 2005). In addition, this well-known distance and motion of the LMC allow for accurate calculations of the total energy that has been released from supernova remnants (SNRs;

^E-mail: m.filipovic@uws.edu.au
Maggi et al. 2016, 2019; Bozzetto et al. 2017; Leahy et al. 2019; Yew et al. 2021). A detailed optical study of 715 LMC Planetary Nebulae (PNe) is presented by Reid \& Parker (2013) and Reid (2014). To date, 31 of these objects have been detected at radio wavelengths by Payne et al. (2008), Filipović et al. (2009), Vukotić et al. (2009), and Leverenz et al. (2017) with 28 having a complete radio-surfacebrightness-to-diameter $\left(\sum-D\right)$ relation derived from $6 \mathrm{~cm}$ surveys. Each of these objects has been observed at multiple radio wavelengths from 3 to $20 \mathrm{~cm}$. Since the LMC is seen almost face-on from the $\mathrm{MW}$, it is a perfect laboratory for studying complete populations with accurate energy measurements (Riess et al. 2019).

The last few decades have seen extensive multifrequency surveys of the sky in the direction of these two galaxies, primarily investigating objects within the MCs themselves. These various surveys present a prime opportunity to study not just intrinsic MC objects but also a large population of background active galactic nuclei (AGNs) and quasars located in the sky area around the LMC. This massive collection of data allows for a high-level analysis of the AGN population with reasonable spectral resolution, sensitivity, and spatial resolution.

Large scale radio continuum surveys by Filipovic et al. (1996b,1998b, c) allowed for a detailed overview of H II and star- 


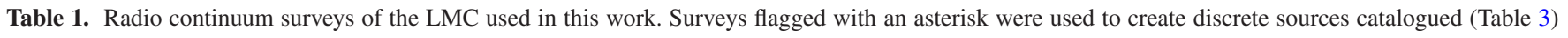
and to estimate spectral index. $\dagger$ indicates that the field is restricted to the $1.384 \mathrm{GHz}$ image size.

\begin{tabular}{|c|c|c|c|c|c|c|c|c|}
\hline Survey & Telescope & $\begin{array}{l}\text { Freq. } \\
(\mathrm{GHz})\end{array}$ & $\begin{array}{l}\text { Field Size } \\
(\operatorname{deg} \times \operatorname{deg})\end{array}$ & $\begin{array}{c}\text { rms } \\
\left(\mathrm{mJy} \mathrm{beam}^{-1}\right)\end{array}$ & $\begin{array}{c}\text { Synthesized } \\
\text { FWHM (arcsec) }\end{array}$ & $\begin{array}{l}\text { Mean Maj. and Min. } \\
\text { Source Size }(\operatorname{arcsec})\end{array}$ & $\begin{array}{l}\text { Num. of } \\
\text { Sources }\end{array}$ & Reference \\
\hline GLEAM* & MWA & 0.200 & $10.8 \times 12.3 \dagger$ & $17-20$ & $162 \times 139$ & $171 \times 146$ & 1277 & This Work \\
\hline $\mathrm{MRC}^{*}$ & MOST & 0.408 & $10.8 \times 12.3 \dagger$ & $18-60$ & $156 \times 172$ & N/A & 40 & Large et al. (1981) \\
\hline SUMSS* & MOST & 0.843 & $10.8 \times 12.3 \dagger$ & $\sim 1.2$ & $45 \times 47$ & $54.27 \times 46.98$ & 3636 & Mauch et al. (2003) \\
\hline ASKAP-Beta & ASKAP & 0.843 & $9.5 \times 9.8$ & 0.71 & $61 \times 53$ & $66.81 \times 56.73$ & 1995 & This Work \\
\hline MOST & MOST & 0.843 & $7.7 \times 7.9$ & $0.30-0.40$ & $45 \times 47$ & $48.90 \times 44.19$ & 2084 & This Work \\
\hline ATCA* & ATCA & 1.384 & $10.8 \times 12.3$ & 0.50 & 40 & $46.98 \times 41.26$ & 6434 & This Work \\
\hline ATCA* & ATCA & 4.80 & $6.0 \times 6.3$ & 0.28 & 33 & $40.97 \times 34.89$ & 570 & This Work \\
\hline PMN & Parkes & 4.85 & $10.8 \times 12.3 \dagger$ & $\sim 8$ & 252 & $312 \times 254$ & 191 & Griffith \& Wright (1993) \\
\hline ATCA* $^{*}$ & ATCA & 8.64 & $6.0 \times 6.3$ & 0.50 & 20 & $25.64 \times 21.70$ & 279 & This Work \\
\hline AT20G* & ATCA & 19.904 & $10.8 \times 12.3 \dagger$ & $\sim 10$ & 13.9 & N/A & 38 & Murphy et al. (2010) \\
\hline
\end{tabular}

forming regions intrinsic to the MCs. 469 discrete sources in the direction of the LMC were detected using the Parkes telescope between 1.4 and $8.55 \mathrm{GHz}$ (Filipovic et al. 1995). Of these, 209 have been classified as H II regions or SNRs. This analysis has allowed for a detailed understanding of the overall structure of the LMC and SMC. Additionally, the detection of these objects allowed cross identification with infrared (IR) surveys to investigate the radio-to-IR comparison (Filipovic et al. 1998a). Most recently, For et al. (2018) presented a survey of the MCs using a low-frequency wideband telescope, the Murchison Widefield Array (MWA; Wayth et al. 2015).

Large background source catalogues have a two-fold significance: (1) they enable population studies to understand the evolution of radio objects and by - extension - the evolution of the Universe, and (2) they allow for the study of foreground media, through Faraday rotation imparted on polarized sources. These Faraday rotation measurements further improve the understanding of the magnetic field strength and direction as measured by Haynes et al. (1991). Building on this, Gaensler et al. (2005), Mao et al. (2012), and Kaczmarek et al. (2017) utilized these background catalogues to more accurately determine the magnetic field strength within the LMC.

From this wealth of data, combined with new surveys and observations, we can further understand the evolution and composition of the AGN population. In this paper, we describe the process of source finding and cross-matching these various source lists to construct a large catalogue of radio sources in the direction of the LMC. We examine each source to determine its radios properties. Our new catalogue presented here is best compared and similar to the Australia Telescope Large Area Survey (ATLAS, at $1400 \mathrm{MHz}$; Norris et al. 2006; Middelberg et al. 2008) that covered a total of $\sim 6 \mathrm{deg}^{2}$ on the sky, down to a root mean squared (rms) noise level of $<30 \mu \mathrm{Jy}$. The ATLAS survey uncovered over 3000 distinct radio sources out to a redshift of 2. Our similar resolution and increased sky coverage will serve to significantly increase our knowledge of these distant objects.

\section{OBSERVATIONAL DATA}

We have used a number of radio continuum images from archival data to detect discrete sources in the direction of the LMC. These data have been taken with the MWA, Australian Square Kilometre Array Pathfinder (ASKAP), the Molonglo Observatory Synthesis Telescope (MOST), and the Australia Telescope Compact Array (ATCA) telescopes (details are shown in Table 1). These observations were originally used to detect and categorize sources and regions intrinsic to the LMC. Below, we describe each of the data sets that we used in this study.

\subsection{MWA images}

Low-frequency surveys are becoming increasingly important in the understanding of objects such as young SNR, Compact Steep Spectrum (CSS), and Gigahertz Peak Spectrum (GPS) sources. In particular, this is important for GPS sources as the turnover can be more accurately identified at this end of the radio frequency bandwidth. As part of the GaLactic Extragalactic All-sky MWA (GLEAM) survey (Wayth et al. 2015), For et al. (2018) presented a series of selected images of the MCs. We used the $0.2 \mathrm{GHz}$ (robust $=-1$ ) image, which has the lowest noise and best angular resolution for this study of individual sources (see Table 1).

\subsection{ASKAP-Beta image}

In this paper, we present a new $0.843 \mathrm{GHz}$ image from the ASKAP Telescope Boolardy Engineering Test Array (BETA; Hotan et al. 2014) that covers a $9.5^{\circ} \times 9.8^{\circ}$ area of the LMC field. This observation was performed in 2015 May as an early ASKAP testing field with a bandwidth of $1 \mathrm{MHz}$. At this time, ASKAP was operated as a 6 antenna interferometer using first generation Phased Array Feed (PAF) receivers. Each receiver produced nine independent beams that were arranged on the sky as a $3 \times 3$ grid with a grid spacing of 1.46 degrees. The extent of the LMC was covered by mechanically re-pointing the antennas at four field centres to reposition the beam pattern on the sky. To increase the density of the mosaic pattern, an additional four field centres were observed, each of which was offset from the original field centre by 0.73 degrees in both RA and Dec. (see Heywood et al. 2016 for a more detailed description).

The observations cycled through these eight field centres approximately once per hour for $10 \mathrm{~h}$, producing the equivalent of a 72pointing mosaic with a traditional, single-beam instrument. These data from each of the 72 beam/field combinations were processed independently using the ASKAPSOFT software package and pipeline. The bandpass and flux density were calibrated using a separate observation of PKS B1934-638. This source was observed at the centre of each of the 9 PAF beams, and calibrated against the Reynolds (1994) model. These data for each beam/field combination were then imaged and self-calibrated independently and the final images were merged into a linear mosaic. From this, we achieve an average rms noise level of $0.71 \mathrm{mJy}^{\text {beam }}{ }^{-1}$ and a full width at half-maximum $(\mathrm{FWHM})$ of $61 \times 53$ arcsec.

Note that our ASKAP-Beta image was made at a very early stage of the ASKAP testing. As expected, a range of issues such as positional accuracy, beam forming accuracy, and calibration were discovered. We have made every effort to identify and correct these problems. 
These efforts have subsequently contributed to understanding and improving the performance of ASKAP generally. As a result, a newer higher sensitivity and resolution ASKAP survey at $888 \mathrm{MHz}$ was recently produced (Pennock et al. 2021). This new survey is built upon results presented here.

\subsection{MOST 0.843 GHz image}

To further enhance our frequency coverage of sources in the direction of the LMC, we use the $0.843 \mathrm{GHz}$ image from the MOST using observations from 1981 that pre-dates the Sydney University Molonglo Sky Survey (SUMSS) (Section 2.6.2) as described by Mills \& Turtle (1984). This image reaches an rms of between 0.3 and $0.6 \mathrm{mJy}_{\text {beam }}{ }^{-1}$ with a resolution of 43 arcsec and a bandwidth of $3 \mathrm{MHz}$. This survey was initially constructed to maximize detection of SNRs and HII regions within the MCs. In this study, however, we decided to use only SUMSS as a core catalogue (also see Section 3.4.3) to be used for the source spectral index investigation as we found the MOST flux densities to be unreliable, especially for the fainter sources.

\subsection{ATCA $1.384 \mathrm{GHz}$ image}

Radio continuum emission at $\sim 1.4 \mathrm{GHz}$ is produced from a variety of sources on all scales. Typically, the use of only one telescope (or array configuration) is not sufficient to fully sample all spatial scales. To counteract this, we have chosen to use the $1.384 \mathrm{GHz}$ images from Hughes et al. (2006, 2007) and Payne et al. (2009) who combine the ATCA and Parkes telescopes LMC surveys (Haynes et al. 1991; Filipovic et al. 1995, 1996b, 1998b). The ATCA Telescope observations were undertaken with four different array configurations providing 40 unique baselines, with a maximum baseline of $750 \mathrm{~m}$. This allows for high-resolution observations to be combined with zero spacing (single-dish) data. Once combined, the final image has an FWHM of 40 arcsec with an rms of $\sim 0.5$ mJy beam $^{-1}$. The data from these two telescopes were taken between 1994 and 1996, covering a $10.8^{\circ} \times 12.3^{\circ}$ area. This field fully covers the LMC with sufficient room beyond the extent of the diffuse emission of the LMC, allowing for a detailed study of various source types to be undertaken.

\subsection{ATCA 4.8 and 8.64 GHz images}

Dickel et al. (2005) observed the LMC using the ATCA's dual band observing mode; one $128 \mathrm{MHz}$ band was placed at a central frequency of $8.64 \mathrm{GHz}$ and the second at a central frequency of $4.8 \mathrm{GHz}$. The main science goal for this survey was to observe and identify SNRs and H II regions that reside within the LMC. These data were combined with observations made with the Parkes telescope (Haynes et al. 1991; Filipovic et al. 1995, 1996b, 1998b) to allow the broad, diffuse radio structure of the LMC to be analysed. 7085 separate ATCA pointing centres were used to cover the $6^{\circ} \times 6^{\circ}$ area, with additional sampling around the 30 Doradus (30 Dor) region. With angular resolutions of $33 \operatorname{arcsec}($ at $4.8 \mathrm{GHz}$ ) and $20 \operatorname{arcsec}$ (at $8.64 \mathrm{GHz}$ ), images were created to be sensitive to the target objects and at the same time closely matching the then-existing X-ray, optical and IR surveys. The resolution from the 352-m and 367-m arrays (configured by excluding the sixth antenna) presents an issue of poor resolution (>1 arcmin) so that sources could not easily be distinguished as background objects or objects within the LMC. From this, an rms sensitivity of $0.28 \mathrm{mJy}^{\text {beam }}{ }^{-1}$ and $0.5 \mathrm{mJy}$ beam ${ }^{-1}$ was achieved for the 4.8 and $8.64 \mathrm{GHz}$ images, respectively.

\subsection{Supplementary surveys}

We make use of an additional four radio surveys and five optical catalogues as summarized in Tables 1 and 2, respectively. These supplementary surveys allow for radio continuum spectra to be sampled over the range of 0.408 to $20 \mathrm{GHz}$, to detect sources with curved or peak radio spectra. Additionally, the inclusion of the SUMSS catalogue provides $0.843 \mathrm{GHz}$ data for the full ATCA $1.384 \mathrm{GHz}$ field. We further include optical surveys to obtain redshift measurements for a small subset of objects.

\subsubsection{Molonglo Reference Catalogue of Radio Sources}

The Molonglo Reference Catalogue of Radio Sources (MRC; Large et al. 1981) was established using the MOST telescope between 1968 and 1974, detecting 12141 sources brighter than $0.7 \mathrm{Jy}$ at $408 \mathrm{MHz}$. This was achieved with an rms of 18-60 $\mathrm{mJy} \mathrm{beam}^{-1}$. The total survey area covers $7.85 \mathrm{sr}\left(\sim 25770\right.$ degrees $\left.^{2}\right)$, with an overall source density of $1500 \mathrm{sr}^{-1}\left(\sim 0.456\right.$ per 1 degree $\left.^{2}\right)$. The catalogue is reasonably complete above $\sim 1 \mathrm{Jy}$, however, the flux measurements between 0.7 and $1 \mathrm{Jy}$ may be unreliable.

\subsubsection{The Sydney University Molonglo Sky Survey}

The SUMSS (Bock, Large \& Sadler 1999; Mauch et al. 2003) was completed in 2007 using MOST and covering the Southern sky south of $-30^{\circ}$ with $|b|> \pm 10^{\circ}$. This survey was designed as an extension of the NRAO VLA Sky Survey (NVSS) on the same grid and at a similar resolution. However, the SUMSS Survey observed at $0.843 \mathrm{GHz}$ with a bandwidth of $3 \mathrm{MHz}$ compared to the $\sim 50 \mathrm{MHz}$ bandwidth of the $1.4 \mathrm{GHz}$ NVSS survey. These two surveys, SUMSS and NVSS, overlap in the region $-40<$ Dec $<-30^{\circ}$ and catalogues have been published that contain 210412 and 1773484 sources, respectively (Condon et al. 1998; Mauch et al. 2003). While the rms noise level of SUMSS $\left(\sim 1 \mathrm{mJy}^{\text {beam }}{ }^{-1}\right)$ is about twice that of NVSS, the $u-v$ coverage for SUMSS is superior to NVSS, allowing for extended objects with lower surface brightness to be detected.

\subsubsection{The Parkes-MIT-NRAO (PMN)}

The Parkes-Massachusetts Institute of Technology (MIT)-National Radio Astronomy Observatory (NRAO) Survey of the sky South of Dec. $=+10^{\circ}$ was performed in 1990 using the Parkes Radio Telescope outfitted with the NRAO seven beam receiver at $4.850 \mathrm{GHz}$ (Griffith \& Wright 1993) with FWHM of 252 arcsec. 36640 sources across the Southern sky were detected down to a $5 \sigma$ level with an rms of $>4.2 \mathrm{mJy}$ beam $^{-1}$. As for the SUMSS, we make use of this catalogue but not of the images.

\subsubsection{The Australia Telescope $20 \mathrm{GHz}$ Survey}

The Australia Telescope $20 \mathrm{GHz}$ Survey (AT20G) is a blind survey that looks for objects above a given rms anywhere in the given sky patch. It was conducted at $19.904 \mathrm{GHz}$ using a bandwidth of $248 \mathrm{MHz}$ that surveyed the entire Southern sky at Dec. $<0^{\circ}$. The primary objective was to categorize and analyse radio populations at high frequencies on an unprecedented scale. Murphy et al. (2010) have presented a catalogue of 5890 sources detected at a flux density level of at least $40 \mathrm{mJy}$ with a 91 percent completeness above $100 \mathrm{mJy}$ for declination south of $-15^{\circ}$, and 79 per cent above $50 \mathrm{mJy}^{\text {beam }}{ }^{-1}$ (some data were lost due to weather for declinations above $-15^{\circ}$ ). 
Table 2. Optical surveys of the LMC used in this work.

\begin{tabular}{lccccl}
\hline Survey & Telescope & $\begin{array}{c}\text { Unique } \\
\text { Source } \\
\text { Count }\end{array}$ & $\begin{array}{c}\text { Matched } \\
\text { Sources }\end{array}$ & $\begin{array}{c}\text { Matched } \\
\text { Sources } \\
\text { with z }\end{array}$ & \multicolumn{2}{c}{ Reference } \\
\hline 6dF & 1.2-m UK Schmidt Telescope & 540 & 111 & 84 & Jones et al. (2009) \\
MACHO & 50” Mt. Stromlo Telescope & 3407 & 31 & 27 & Kim et al. (2012) \\
Magellanic Quasars Survey. I & Anglo-Australian Telescope & 4679 & 122 & 3 & Kozłowski \& Kochanek (2009) \\
Magellanic Quasars Survey. II & Anglo-Australian Telescope & 677 & 10 & 0 & Kozłowski et al. (2012) \\
Magellanic Quasars Survey. III & Anglo-Australian Telescope & 565 & 20 & 20 & Kozłowski et al. (2013) \\
\hline
\end{tabular}

\subsubsection{6dF Galaxy Survey}

The 6 degree field (6dF) Galaxy Survey (Jones et al. 2005a, b) is an optical survey conducted with the 1.2-m UK Schmidt Telescope with the goal of obtaining spectroscopy for a magnitude limited sample of galaxies to $\left(\mathrm{K}, \mathrm{H}, \mathrm{J}, r_{F}, b_{J}\right)=(12.75,13.00,13.75,15.60,16.75)$. This was achieved with the $6 \mathrm{dF}$ instrument, using 150 spectroscopic fibres positioned by a robot on the focal plane. From this, 136304 redshifts of galaxies were measured for galactic latitudes $|b|>10 \mathrm{deg}$, over an area of $17000 \mathrm{deg}^{2}$. Each source in the $6 \mathrm{dF}$ catalogue was selected from the Two Micron All Sky Survey Extended Source Catalogue (2MASX) catalogue (Jarrett et al. 2000; Skrutskie et al. 2006) $\left(K_{\text {tot }}\right.$ $<12.75)$. A near-IR selection has a two-fold effect: (1) the target sample is less affected by dust extinction; (2) typically, candidates selected solely through optical means will be dominated by young, blue stellar populations. However, candidates chosen through nearIR criteria are dominated by their oldest population of stars, allowing for more robust galactic mass measurement, and are more likely to host an AGN.

\subsubsection{Magellanic Quasars Survey (MQS)}

Kozłowski \& Kochanek (2009), Kozłowski, Kochanek \& Udalski (2011), Kozłowski et al. (2012, 2013) classified a total of 6103 optical sources in the direction of the LMC as Quasi-Stellar Object (QSO)'s using Australian Astronomical Telescope. Of these sources, 962 have redshift measurements. Crucially, these sources were selected using multiple methods to confirm that they do not belong to the LMC. Each source was inspected for its mid-IR colours compared against existing optical surveys, with the Spitzer SAGE survey (Meixner et al. 2006) and the OGLE-III survey (Udalski et al. 1994). The redshift measurements presented in these surveys are of great importance as they allow us to disentangle sources within the LMC against the background AGN. Additionally, these surveys allow for the ease of cross-matching radio sources to the CatWISE2020 IR source catalogue (as shown in Section 2.6.8).

\subsubsection{MACHO survey}

The Massive Astrophysical Compact Halo Objects (MACHO) survey, as presented by Kim et al. (2012), provides measurements of 663 QSO candidates in the direction of the LMC with the intent of searching for MACHO. These sources were cross-matched with additional surveys, such as Spitzer SAGE, Two Micron All Sky Survey (2MASS), Chandra, the XMM-Newton and an LMC UBVI catalogues. The authors applied machine learning techniques to these data, allowing for a high confidence classification of each source. This gives an estimated false positive rate of 1 per cent for cross identification with other surveys. This allows us to identify sources in our radio catalogue with QSO candidates from Kim et al. (2012).

\subsubsection{CatWISE2020 catalogue}

The Wide-Field Infrared Survey Explorer (WISE) space telescope has mapped the whole sky at wavelengths of 3.4, 4.6, 11.6, and $22 \mu \mathrm{m}$ (hereafter W1, W2, W3, and W4) as presented by Wright et al. (2010). From this, the CatWISE2020 catalogue was created (Marocco et al. 2021) where all existing data products from all phases were combined to make the most sensitive all-sky mid-IR survey to date. In total, two complete and 10 additional all sky passes were made (data collected from 2010 to 2018), allowing for identification and rejection of asteroids and other Solar system objects. ${ }^{1}$ This survey was conducted until the telescope ran out of liquid helium, pushing the rms significantly higher than initially designed. Recently, Marocco et al. (2021) produced a catalogue of over 1890715640 entries for the W1 and W2 bands.

\section{RADIO SOURCE CATALOGUE}

We generated five new source lists of discrete radio-continuum sources from the 0.843 (ASKAP-Beta), 0.843 (MOST), 1.384, 4.8, and $8.64 \mathrm{GHz}$ ATCA images. When combined with the existing SUMSS, a total of 6434 unique discrete radio sources have been detected in at least one of these catalogues. The source finding for each image was performed as a blind search without the use of prior knowledge from any existing catalogues.

Sources detected in these lists are a combination of sources belonging to the LMC (such as SNRs, H II regions, or PNe) and background objects in the direction of the LMC. By cross-matching (radius of 10 arcsec) with objects in SIMBAD Database (Wenger et al. 2000), no known objects from our Galaxy were detected in any of the previous radio surveys of this area that we study here in this paper. Galactic radio sources are expected to have a low surface density in the direction of the LMC due to its high Galactic latitude (Filipovic et al. 1998b).

\subsection{Source finding and cross-matching}

The AEGEAN Source Finding suite of tools (Hancock et al. 2012a, b; Hancock, Trott \& Hurley-Walker 2018) have been used to process the core radio surveys, creating a catalogue of the sources within the field, including source positions, sizes, and integrated flux densities. A two-step process was applied to the images for source detection, first forming a background and rms map, and subsequently running the source finding routine. The background and rms maps were created with The Background And Noise Estimation tool (BANE) using a moving box-car smoothing method. Using this rms map improves the integrity of the flood fill operation used in AEGEAN as it is able to

\footnotetext{
${ }^{1}$ http://wise2.ipac.caltech.edu/docs/release/neowise/neowise_2019_release_i ntro.html
} 


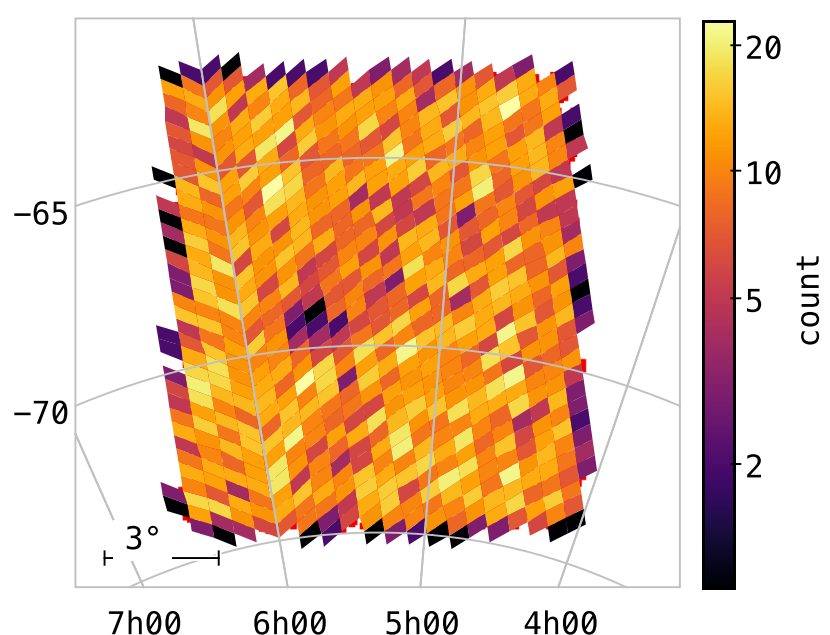

Figure 1. A map of the source counts in HEALPix pixel map in equatorial coordinate system ( $x$-axis is RA J2000 and $y$-axis is Dec. J2000). The map is produced using TOPCAT with HEALPix level 7 (pixel area is $\approx 0.21 \mathrm{deg}^{2}$ ). As scale bar shows, black coloured pixel indicate low density source population which is in agreement with intentionally avoided fields such as the 30 Dor and outer edge of the survey where our coverage is limited.

more accurately determine the source extent, and thus the integrated flux density of the source. For both AEGEAN and BANE, we set tuning parameters to their default values. The cross identification and visual analyses were performed with the Tool for OPerations on Catalogues And Tables (TOPCAT; Taylor 2005, 2011) and SAOIMAGE DS9 (DS9) applications (Joye \& Mandel 2003).

We used the following five steps to create the final source lists presented in this paper:

(i) Initially, we began with a $5 \sigma$ detection level, creating preliminary source lists from the 0.843 (ASKAP and MOST images), 1.384, 4.8, and $8.64 \mathrm{GHz}$ images.

(ii) We manually examined sources that were close to $\mathrm{S} / \mathrm{N} \sim 5$ and $\theta_{\text {major }} / \theta_{\text {minor }}>2$ to determine if they were spurious. Detections that were not genuine (e.g. due to imaging artefacts, or an increased noise level towards the field edge as evident in Fig. 1) were flagged and removed.

(iii) We visually inspected large, extended sources belonging to the LMC using the SIMBAD Database and HASH PN database (Parker, Bojičić \& Frew 2016, hashpn.space) to identify known SNRs, PNe, and $\mathrm{H}$ II regions (Filipovic et al. 1998c). The SNRs and PNe found here are addressed in Filipović et al. (2009) and Bozzetto et al. (2017) and are excluded together with all known H II regions from the catalogue presented in this paper.

(iv) The ATCA $1.384 \mathrm{GHz}$ source list was taken as the starting point which for the cross-match with the other source lists extracted from the above listed radio surveys. We use a nearest neighbour matching radius of 30 arcsec to determine whether there was a detection at any other frequencies. With a secondary internal match we were able to identify sources that have multiple components at the higher frequencies of 4.8 and $8.64 \mathrm{GHz}$, while being unresolved at the lower frequencies, 0.843 and $1.384 \mathrm{GHz}$. After each crossmatch, a best match position was created for each source to aid in subsequent cross-matching. This best-match position is taken from the highest resolution component for the source.

(v) A second and final, targeted source finding sweep was conducted on all images using prior knowledge from three ATCA source lists. We also apply this prior knowledge where the source does not have a detection above $5 \sigma$ for the image in question. Consequently, this allows for a second source finding pass to detect sources down to $3 \sigma$ and cross-matching (and merging) these into the existing source lists.

We estimate a false cross-match rate of $\sim 1$ percent by shifting each source list in RA by three times the cross-matching criterion. For example, the $4.8 \mathrm{GHz}$ to $1.384 \mathrm{GHz}$ cross-match radius is 30 arcsec, hence we shift the $4.8 \mathrm{GHz}$ image northward by 90 arcsec and repeat the cross-matching procedure. This removes any real matches so that we are left with only random matches.

Supplementary source lists have been added to more accurately constrain the spectral index of each source as described in Section 2.6. AT20G was the first of these supplementary source lists to be crossmatched with all of the preceding surveys. The addition of this source list reveals a limited number of sources (38) but provides a significant increase in the positional accuracy and a wider frequency range which is essential to a better constrained source spectral energy distribution (SED). The best-match position was then replaced with the position from the highest angular resolution survey. In line with this, the next supplementary source list to be added was the SUMSS catalogue, as it had the next best resolution. Similarly, the best match position was recalculated before repeating this cycle with the MWA, MRC, and PMN source lists. The PMN catalogue was cross-matched with a 252 arcsec search radius due to its poor resolution compared to our other surveys. Unlike for the four core radio surveys (SUMSS at $0.843 \mathrm{GHz}$ and ATCA at $1.384,4.8$, and $8.64 \mathrm{GHz}$ ), a further internal cross-match was not performed on these supplementary surveys.

Multiple cross-matches of a source may be present throughout each of the source lists that have been acquired from existing surveys or generated through AEGEAN. This is due to the differing resolutions of each image, allowing for one detection to be resolved into multiple detections at higher frequencies. These sources have been flagged in our source lists and catalogued as 'Confused Source'. Sources of this nature have been excluded from the following analysis, because of their complexity.

Care must be taken when cross-matching these detections due to the differing resolutions and positional accuracies, both of which create false matches. These false matches and erroneous data points can be potentially corrected by understanding trends in the crossmatch between different pairs of catalogues as shown in Sections 3.2, 3.3, and 4.3.1. Once identified, a manual approach (source-by-source visual inspection) is used to investigate the erroneous properties bf such as flux density, spectral index, and exact position. This cycle of analysis is vital in creating a reliable catalogue.

Additionally, extensive cross checking with the Magellanic Cloud Emission Line Survey (MCELS) in $\mathrm{H} \alpha$, [S II], and [O III] images presented by Smith \& MCELS Team (1998) has been performed to identify sources in both the optical and radio bands. These matches are most likely intrinsic to the LMC (see e.g. Yew et al. 2021). Radio sources with optical counterparts as detected in MCELS have been excluded from further analysis in this work. We note that the MCELS images have been continuum subtracted and no compact stellar optical sources can be detected. Hence, these objects detected in the MCELS images are likely to have an LMC origin and will be studied in our subsequent papers. Also, we make use of other existing optical catalogues that have known background sources, as determined from their redshifts (discussed in Section 4). 98 such radio sources from the MCELS have extended emission (optically diffuse objects) while 129 sources have a compact optical object at or near the centre of the radio source. An additional cross check was made with known objects, such as $80+$ SNRs, $20+$ PNe, and 
Table 3. Example of the point source catalogue of 6434 objects in the directions of the LMC with its positions, integrated flux densities with associated uncertainty, and spectral index. The columns provided are as follows: (1) Source name derived from the highest frequency source component; (2) position of J2000 right ascension from the highest frequency detection; (3) position of J2000 declination from the highest frequency detection; (4) flux density flag for the GLEAM survey; (5 and 6) GLEAM MWA $200 \mathrm{MHz}$ survey integrated flux density; (7 and 8) SUMSS $843 \mathrm{MHz}$ survey integrated flux density; (9) flux density flag for the $20 \mathrm{~cm}$ ATCA $1.384 \mathrm{GHz}$ survey; (10 and11) $20 \mathrm{~cm}$ ATCA $1.384 \mathrm{GHz}$ survey integrated flux density; (12 and 13) $6 \mathrm{~cm}$ ATCA $4.85 \mathrm{GHz}$ survey integrated flux density; (14 and 15) $3 \mathrm{~cm}$ ATCA $8.64 \mathrm{GHz}$ survey integrated flux density; (16 and 17) MRC $408 \mathrm{MHz}$ survey integrated flux density; (18 and 19) PMN 4.8 GHz survey integrated flux density; (20 and 21) AT20G survey integrated flux density at (20 and 21) $4.8 \mathrm{GHz}$; (22 and 23) $8.6 \mathrm{GHz}$; (24 and 25) $19.904 \mathrm{GHz}$; (26 and 27) spectral index fit to all available source components with the associated error; (28) number of flux densities used in the spectral index fit and (29) source classification. Upper limits and uncertain values in flux densities flag columns are designated with preceding < and colon (:), respectively. The columns not presented in this table but available in the full version are: flux densities from MRC, PMN, and AT20G surveys. The full catalogue is provided through the VIZIER service and as supplementary material.

\begin{tabular}{|c|c|c|c|c|c|c|c|c|c|c|}
\hline $\begin{array}{l}\text { (1) } \\
\text { Name J }\end{array}$ & $\begin{array}{c}(2) \\
\text { RA } \\
(\text { J2000) } \\
\text { h m s }\end{array}$ & $\begin{array}{c}(3) \\
\text { Dec. } \\
(\mathrm{J} 2000) \\
0^{\prime}, "\end{array}$ & $\begin{array}{c}(4,5, \text { and } 6) \\
S_{\mathrm{MWA}} \\
0.200 \mathrm{GHz} \\
(\mathrm{mJy})\end{array}$ & $\begin{array}{c}(7 \text { and } 8) \\
S_{\text {SUMSS }} \\
0.843 \mathrm{GHz} \\
(\mathrm{mJy})\end{array}$ & $\begin{array}{c}(9,10, \text { and } 11) \\
S_{20 \mathrm{cmATCA}} \\
1.384 \mathrm{GHz} \\
(\mathrm{mJy})\end{array}$ & $\begin{array}{c}(12 \text { and } 13) \\
S_{6 \mathrm{cmATCA}} \\
4.85 \mathrm{GHz} \\
(\mathrm{mJy})\end{array}$ & $\begin{array}{c}(14 \text { and } 15) \\
S_{3 \mathrm{cmATCA}} \\
8.64 \mathrm{GHz} \\
(\mathrm{mJy})\end{array}$ & $\begin{array}{l}(\ldots) \\
\ldots \\
\ldots \\
\ldots\end{array}$ & $\begin{array}{c}\text { (26 and 27) } \\
\alpha \pm \Delta \alpha\end{array}$ & $\begin{array}{l}(29) \\
\text { Class }\end{array}$ \\
\hline $040217-734215$ & 040217.8 & -734215 & $110 \pm 16$ & $20.9 \pm 1.5$ & $: 13.8 \pm 1.6$ & $\ldots$ & $\ldots$ & $\ldots$ & $-1.09 \pm 0.09$ & \\
\hline 040447-732915 & 040447.2 & -732915 & $<98$ & $10.2 \pm 1.6$ & $: 6.3 \pm 0.9$ & $\ldots$ & $\ldots$ & $\ldots$ & $-0.9 \pm 0.4$ & \\
\hline 040455-732752 & 040455.0 & -732753 & $<98$ & $37.3 \pm 1.8$ & $: 25.4 \pm 2.6$ & $\ldots$ & $\ldots$ & $\ldots$ & $-0.8 \pm 0.2$ & \\
\hline 040456-734144 & 040456.7 & -734144 & $\ldots$ & $\ldots$ & $2.93 \pm 0.5$ & $\ldots$ & $\ldots$ & $\ldots$ & $\ldots$ & \\
\hline$\cdots$ & $\ldots$ & $\ldots$ & $\ldots$ & $\ldots$ & $\ldots$ & $\ldots$ & $\ldots$ & $\ldots$ & $\ldots$ & \\
\hline
\end{tabular}

$100+$ H II regions. Variable sources may be present but are hard to detect as this catalogue has been compiled from data sets with sparse coverage over a long time-scale $(\sim 31 \mathrm{yr})$.

A sample of the final catalogue is shown in Table 3. This table includes our combined selected radio source lists from all surveys apart from two $0.843 \mathrm{GHz}$ lists from ASKAP-Beta and MOST surveys as discussed in Sections 3.4.2 and 3.4.3. The first column of Table 3 is using the standard International Astronomical Union (IAU) source naming format of Jhhmmss-ddmmss (Dickel 2000). This name has been derived from the position of the highest resolution detection for each source from all input source lists. Columns 2 and 3 give the source position from the highest resolution detection of the source. The full catalogue is available through the VizieR Catalogue Service (Ochsenbein, Bauer \& Marcout 2000). For each survey in the final combined catalogue, we provide a source position, size, integrated flux density, peak flux density, and a local rms measure. From this, we calculated a spectral index $(\alpha)$ where possible which is defined as $S_{v} \propto v^{\alpha}$, where $S_{v}$ is flux density at frequency $v$.

\subsection{Selection effects and completeness}

Our catalogue of discrete radio sources presented here is not complete because of various problems including (but not limited to) detection limits, diffuse emission from the LMC, imaging artifacts, variable and changing sensitivity (across the field of a given survey) as well as the resolution limitations for both foreground and background objects. This is most notable in the 30 Dor region where large sidelobes are produced by strong and diffuse emission, especially in our ATCA images. This area has been excluded from the analysis to prevent contamination from this emission and the spurious clean components created during the imaging phase (see Fig. 1). We define this exclusion area around 30 Dor after inspecting the $1.384 \mathrm{GHz}$ and $0.843 \mathrm{GHz}$ images and then we applied this exclusion to all subsequent source finding catalogues. This is the area between RA(J2000) of $5^{\mathrm{h}} 30^{\prime}$ and $5^{\mathrm{h}} 47^{\mathrm{m}}$ and Dec.(J2000) from $-68^{\circ} 50^{\mathrm{m}}$ to $-70^{\circ}$. The rms estimates presented in this work are the best case measurement, i.e. taken from source free regions on the outskirts of the LMC. As mentioned above (Section 3.1), we have excluded all known SNRs and H II regions listed by Bozzetto et al. (2017) and various other surveys covering sources that are a part of the LMC. This has allowed us to have a less contaminated population of background (such as Quasars and AGNs) and foreground objects (mainly Galactic radio stars).

\subsection{Discrete source finding errors}

It is widely accepted that all source finders have an error rate ${ }^{2}$ of at least a few per cent. However, this depends a lot on the threshold settings. The compromise is always between completeness (of real sources) and reliability. AEGEAN is no exception to this, as shown in Hopkins et al. (2015). The catalogue from each image was carefully visually inspected for spurious sources due to image artefacts and regions of strong emission. For example, detections were made along the edge of each image that were either incomplete or false detections, requiring a manual pass to remove these sources. This manual pass was extended to the catalogue as a whole, with a focus on regions of strong emission (either side lobes or extended emission) and sources with unrealistic properties (such as extremely large Gaussians, strong sources, and source positions beyond the limits of the observed area).

\subsection{Discrete source position and integrated flux density uncertainties}

The richness of data available for this field allows us to compare and, if necessary, post-calibrate our new source lists. This was particularly useful for the ASKAP-Beta data, which was re-calibrated against the more reliable SUMSS catalogue.

\subsubsection{ASKAP-beta and MOST survey positional correction}

To determine the positional accuracy of the ASKAP-Beta data set, we have made comparisons with the SUMSS $0.843 \mathrm{GHz}$ source list. From this, we find that the ASKAP-Beta data set requires a $\sim 3$ arcsec correction of its source position to be consistent with existing catalogues. Larger offsets are found when comparing the ATCA

\footnotetext{
${ }^{2}$ Includes the number of false positives and the missing real sources.
} 

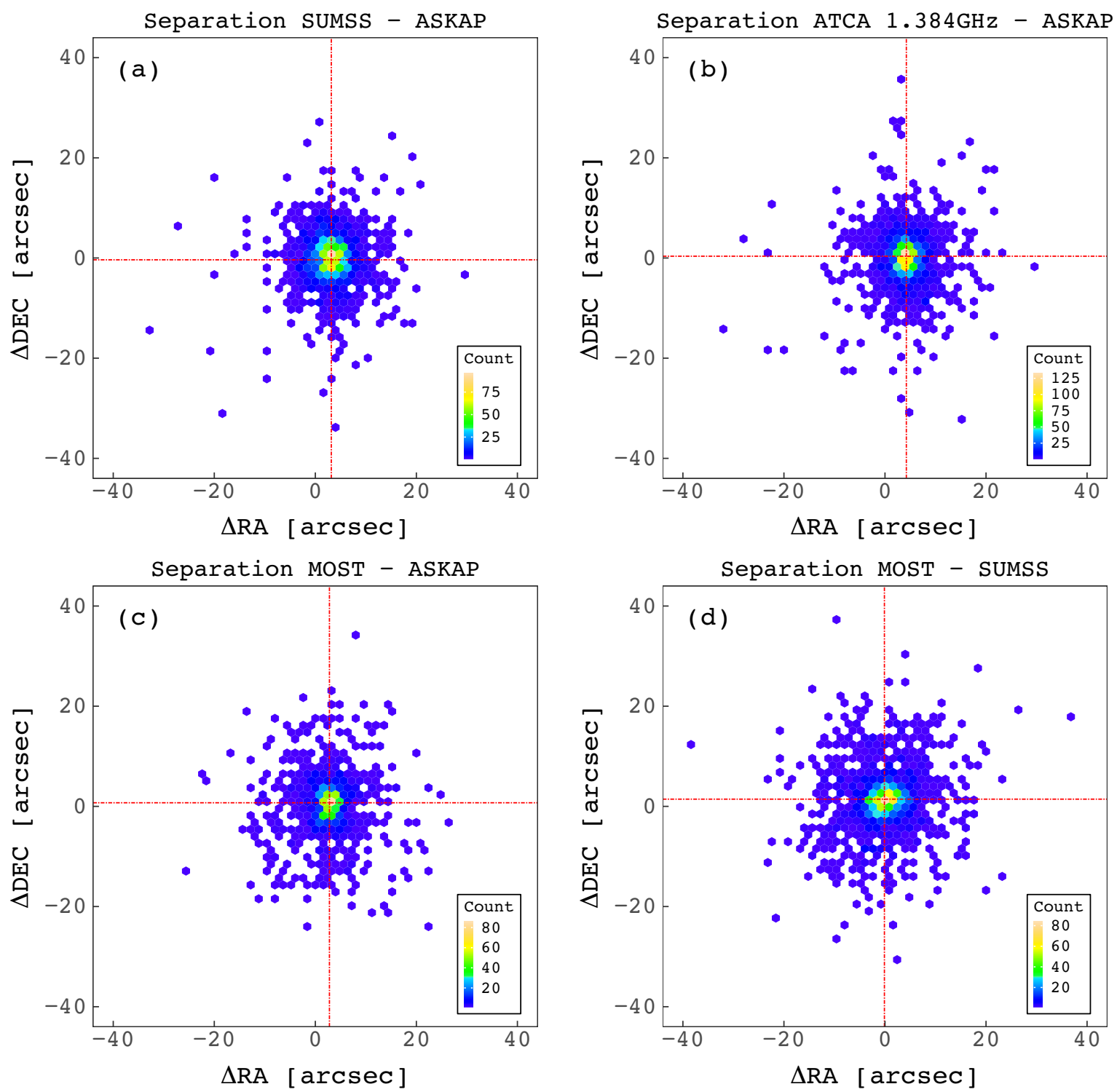

Figure 2. Positional comparisons between (a) SUMSS - ASKAP-Beta, (b) ATCA $1.384 \mathrm{GHz}$ - ASKAP-Beta, (c) MOST - ASKAP-Beta, and (d) MOST SUMSS catalogues without applying ASKAP-Beta positional corrections. The median RA and Dec. offsets are shown with vertical and horizontal red dot-dashed line, respectively. The median positional offsets in RA and Dec., respectively, are (a) 3.18 and -0.36 arcsec, (b) 4.23 and 0.36 arcsec, (c) 3.18 and -0.36 arcsec, and (d) -0.11 and 1.44 arcsec.

$1.384 \mathrm{GHz}$ catalogue with the ASKAP-Beta catalogue as the median positional offset between the two catalogues is 4.23 and 0.36 arcsec in RA and Dec., respectively. A similar discrepancy is identified when positional comparisons are made with the $0.843 \mathrm{GHz}$ MOST source list. The median positional offsets are 2.79 and 0.72 arcsec in RA and Dec., respectively, as shown in Fig. 2. The cross-matching process was performed after correcting the positional error. A comparison of positional offsets between pairs of catalogues is shown in Fig. 2.

\subsubsection{ASKAP-Beta flux density post-calibration}

We make use of the SUMSS survey as an established, external catalogue in order to determine the completeness and quality of our newly produced source lists of ASKAP-Beta and MOST surveys.

It is now understood that the ASKAP-Beta system and, by extension, the observations made during that time had an inaccurate amplitude calibration of the order of $\sim 10-15$ per cent. This affected the flux density measurement and caused an additional positional offset (Section 3.4.1).

A systematic correction was derived from the comparison with the SUMSS survey as no frequency interpolation is required to compare fluxes. Additionally, we have used the SUMSS catalogue to measure the completeness and reliability of the $0.843 \mathrm{GHz}$ MOST source list (as shown in Section 3.2). Using these surveys, we perform a leastsquares linear fit to determine the systematic positional offset present within the ASKAP-Beta data set. Before correcting these data, the ratio of $S_{\text {ASKAP-Beta }} / S_{\text {MOST }}$ for the integrated flux was 0.880 ; similarly the ratio of $S_{\text {ASKAP-Beta }} / S_{\text {SUMSS }}$ was 0.893 . From these results, we have applied a 12.75 percent systematic increase to the ASKAPBeta fluxes.

Even after applying the above corrections, however, at lower flux densities $(<15 \sigma)$ our ASKAP-Beta flux densities still show a large 

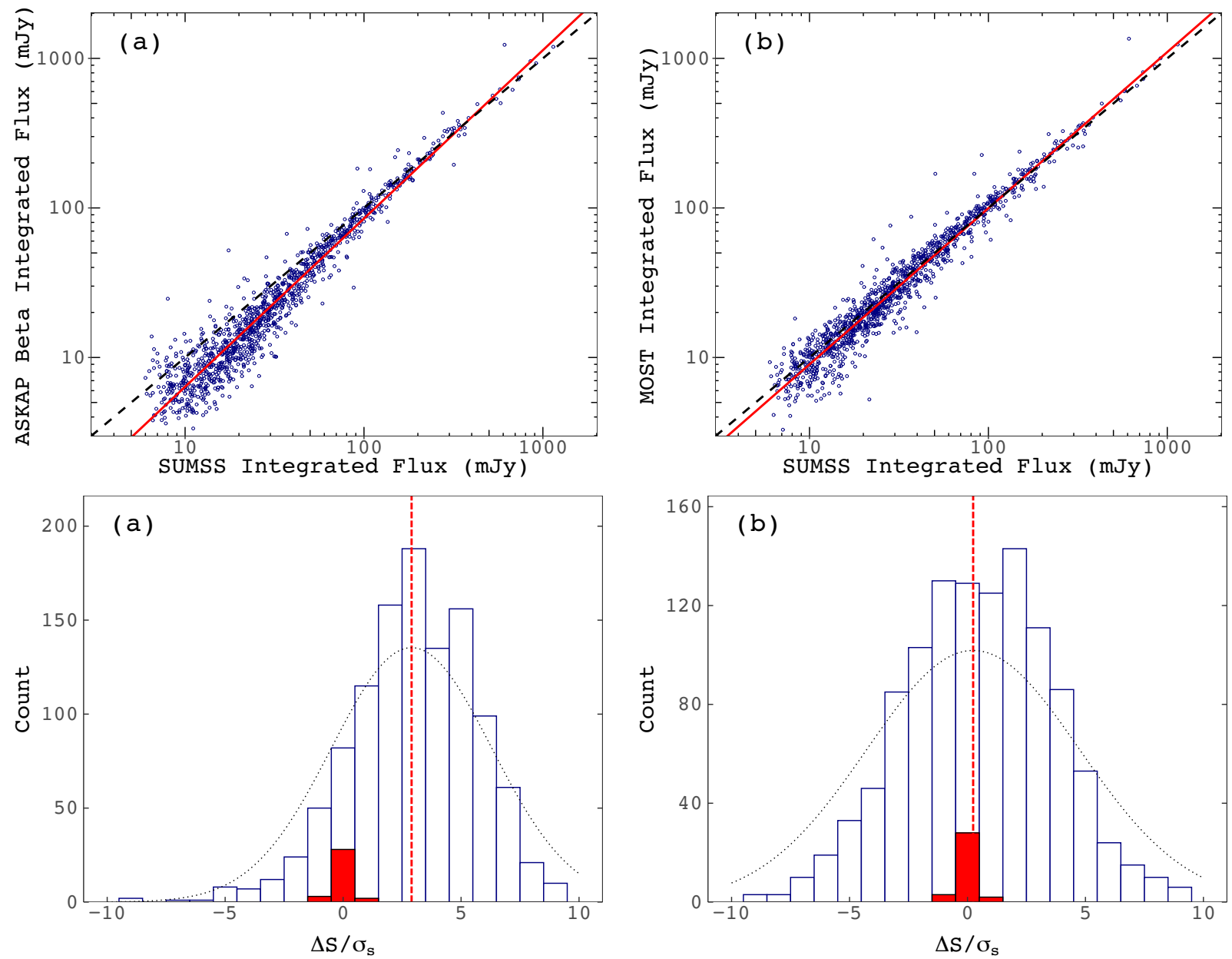

Figure 3. Top row: An integrated flux density comparison of the ASKAP-Beta (a) and MOST (b) $0.843 \mathrm{GHz}$ source lists, with the SUMSS (see Sections 3.4 .2 and 3.4.3 for more details). In each figure, we represent a ratio of 1 with the black dashed line and the best-fitting line is in red. Bottom row: Histogram of flux density differences weighted with the root sum of squares of corresponding uncertainties. Histogram has a bin size of 1 normalized flux difference. Vertical red dashed line represents the mean of the distribution. Dotted line is a Gaussian distribution with the same mean and standard deviation as in the histogram. Red-filled histogram shows the distribution of $\Delta S / \sigma_{s}$ between matched sources from PMN and ATCA (Dickel et al. 2005), respectively, at $4.8 \mathrm{GHz}$.

Table 4. Example for the source list (total of 1995 sources) at $0.843 \mathrm{GHz}$ derived from ASKAP-Beta survey. Note that the flux densities shown are flux corrected (see text for more details). The uncertainties are only from the fitting method, i.e. flux calibration errors have not been included in the presented uncertainty. The full catalogue is provided through the VIZIER service and as supplementary material.

\begin{tabular}{lccccc}
\hline No & $\begin{array}{c}\text { RA (J2000) } \\
\text { h m s }\end{array}$ & $\begin{array}{c}\text { Dec. (J2000) } \\
\text { ( }^{\prime \prime}\end{array}$ & $\begin{array}{c}\text { Peak Flux } \\
\left(\mathrm{mJy} \mathrm{beam}^{-1}\right)\end{array}$ & $\begin{array}{c}\text { Int. Flux } \\
(\mathrm{mJy})\end{array}$ & $\begin{array}{c}\text { Local rms } \\
\left(\mathrm{mJy} \mathrm{beam}^{-1}\right)\end{array}$ \\
\hline 1 & 043507.4 & -682424 & $9.0 \pm 2.0$ & $12.0 \pm 2.0$ & 1.62 \\
2 & 043508.7 & -705908 & $8.6 \pm 0.9$ & $9.0 \pm 1.0$ & 0.9 \\
3 & 043511.7 & -712652 & $17.2 \pm 0.7$ & $23.0 \pm 1.0$ & 0.76 \\
4 & 043518.1 & -704613 & $16.2 \pm 0.9$ & $20.0 \pm 1.0$ & 0.9 \\
5 & 043519.7 & -722109 & $4.9 \pm 0.8$ & $6.0 \pm 1.0$ & 0.79 \\
$\ldots$ & $\ldots$ & $\ldots$ & $\ldots$ & $\ldots$ & $\ldots$ \\
\hline
\end{tabular}

variance. In Fig. 3(a), we show a direct comparison between the estimated flux densities and histograms of the flux density differences normalized to the weighted root sum square of the corresponding uncertainties (see section 4 of Gregory \& Condon 1991). The plots clearly show that the deviation from the expected value is significant and it appears to be a non-linear systematic function of the flux density (i.e. the lower ASKAP fluxes appear to be systematically underestimated). Not just flux scale, but poorly known ASKAP beam shapes are a huge problem. The primary beam correction factors were poorly known and variable at the time of testing.

The resulting source list is provided in Table 4. While we found that this list is useful for the confirmation (and detection) of faint sources, we decided not to use the ASKAP data in the following analysis and in our combined catalogue shown in Table 3. We also note the problem that $S_{\text {int }} / S_{\text {peak }}$ is systematically higher than one, by typically 20 per cent. 
Table 5. Example for the source list (total of 2084 sources) at $0.843 \mathrm{GHz}$ derived from MOST. The uncertainties are only from the fitting method i.e. flux calibration errors have not been included in the presented uncertainty. The full catalogue is provided through the VIZIER service and as supplementary material.

\begin{tabular}{cccccc}
\hline No & $\begin{array}{c}\text { RA (J2000) } \\
\text { h m s }\end{array}$ & $\begin{array}{c}\text { Dec. (J2000) } \\
\text { I' }^{\prime \prime}\end{array}$ & $\begin{array}{c}\text { Peak flux } \\
\left(\mathrm{mJy} \mathrm{beam}^{-1}\right)\end{array}$ & $\begin{array}{c}\text { Int. flux } \\
(\mathrm{mJy})\end{array}$ & $\begin{array}{c}\text { Local rms } \\
\left.\text { (mJy beam }^{-1}\right)\end{array}$ \\
\hline 1 & 042951.8 & -722719 & 42.0 & $43.0 \pm 4.0$ & 1.55 \\
2 & 043046.8 & -721635 & $31.0 \pm 4.0$ & $81.0 \pm 2.0$ & 1.29 \\
3 & 043402.8 & -723630 & 7.0 & $7.6 \pm 0.8$ & 1.42 \\
4 & 043424.1 & -721503 & 14.0 & $14.0 \pm 1.0$ & 0.96 \\
5 & 043454.8 & -723956 & 299.0 & $312.3 \pm 0.7$ & 1.42 \\
$\ldots$ & $\ldots$ & $\ldots$ & $\ldots$ & $\ldots$ & $\ldots$ \\
\hline
\end{tabular}

\subsubsection{MOST survey flux density post-calibration}

The SUMSS survey reaches an $\mathrm{rms}$ of $\sim 1 \mathrm{mJy}^{\text {beam }}{ }^{-1}$ allowing for $\sim 78$ percent (1638 sources) of sources in the $0.843 \mathrm{GHz}$ MOST source list to be cross-matched and compared against the sources within SUMSS. From this, we find that all SUMSS sources have a $0.843 \mathrm{GHz}$ MOST counterpart (within the overlap region). However, not all $0.843 \mathrm{GHz}$ MOST sources within the same limits (rms and spatial) have an SUMSS detection. As expected, we detect an additional 446 sources in the $0.843 \mathrm{GHz}$ MOST image that are undetected in SUMSS.

These 1638 sources common to both lists, allow us to determine the flux density accuracy of our $0.843 \mathrm{GHz}$ MOST sources (as shown in Fig. 3b). The ratio of the integrated flux densities for these two surveys $\left(S_{\text {SUMMS }} / S_{\text {MOST }}\right)$ is $1.02 \pm 0.01$. This ratio does not show any systematic offset between two source lists. In Fig. 3(b) (lower right panel), we can see that the distribution of normalized flux density differences are much wider than acceptable.

As for the ASKAP-Beta flux density analysis, we can use the MOST detections for cross-checking of faint source detections. However, we are not using these flux densities in source spectral index estimates and in constructing our main catalogue Table 3 . We present the MOST $0.843 \mathrm{GHz}$ source list with its positions and all measured flux densities as a separate Table 5 .

\subsubsection{Other flux density errors and uncertainties}

Uncertainties in flux density estimates are mainly a product of gain calibration uncertainties $\left(\sigma_{g}\right.$; usually given as a percentage of the measured flux density $S$ ) and the uncertainty $\left(\sigma_{n}\right)$ caused by fitting in the presence of the local noise. The total uncertainty $\left(\sigma_{\text {tot }}\right)$ can be then calculated as

$\sigma_{\text {tot }}^{2}=\sigma_{n}^{2}+\left(S \cdot \sigma_{g}\right)^{2}$.

We applied a 10 percent uncertainty in the gain $\left(\sigma_{g}\right)$ to our new ATCA flux estimates at $1.384,4.8$, and $8.64 \mathrm{GHz}$, as recommended in Harvey-Smith et al. (2018).

All source finding tools have a degree of error in their flux density estimation (see also Section 3.3). The main source of this inaccuracy is due to the noise present within any image, causing the sources at or near the detection threshold to have large flux density errors as shown in Fig. 4. In addition to this effect, source finding tools can incorrectly determine the extent of a source, and in most cases will overestimate source flux density (Hopkins et al. 2015).

To determine this, we measure the error of the integrated flux density as a function of fitting error $\left(\sigma_{n}\right)$ to find outlier sources that have been fitted poorly, as shown in Fig. 4. As expected, the relative error increases with weaker sources. Statistically, $\sigma_{n}$ for the majority of our sources is less than 15 per cent across all five main catalogues. However, for some smaller portion of sources it can reach up to 30 per cent.

\subsubsection{MWA Survey flux density post-calibration}

The radio images presented by For et al. (2018) were processed by Hurley-Walker et al. (2017) as part of a large analysis of the GLEAM survey to produce an extragalactic catalogue. During the flux density calibration of the final mosaics of the GLEAM survey, a correction was made for the primary beam of the instrument, which was poorly modelled at the time of processing. This was performed by measuring the ratio of flux density of sources between GLEAM and ancillary catalogues in the Northern sky, then assuming symmetry and applying this correction to the Southern sky. At declinations far from the local zenith $\left(\delta \sim-27^{\circ}\right)$ the correction was less accurate, resulting in an estimated flux density calibration accuracy of 8 percent for $-72^{\circ}<\delta<18^{\circ}$ and 13 per cent outside of this range, escalating to $\approx 90$ per cent at the celestial pole.

The GLEAM data comprise a 'wideband' $170-231 \mathrm{MHz}$ image, typically used for source detection, and $20 \times 7.68 \mathrm{MHz}$ 'sub-band' images, covering 72-231 MHz. In the catalogue formed by HurleyWalker et al. (2017), sources detected in the wideband image at peak flux density $>5 \sigma$, where $\sigma$ is the local rms noise, are measured in the sub-band images using the priorized fit function of AEGEAN (Hancock et al. 2018). We used an identical procedure to HurleyWalker et al. (2017) to produce a catalogue for this region of sky, including accurate rms, background, and PSF measurement. Note that from these multifrequency data, SEDs can be measured for brighter sources, enabling a more accurate flux density measurement, but for fainter sources the spectra are biased towards being flat by the uncertainties, and the wideband images provide the best measurement of the integrated source flux densities, which we here label as $S_{200 \mathrm{MHz}}$ GLEAM.

The high-frequency data presented in this paper enable us to test the flux density scale accuracy and recalibrate the GLEAM data. We first selected sources which would not be confused in the MWA data by excluding any sources within 2 arcmin of any other source, yielding a catalogue of 5419 sources. For each source, we fitted a weighted linear power-law SED using the scipy.stats.leastsq implementation of the Levenberg-Marquardt algorithm to all of the high-frequency data. From these fitted spectra, we predicted

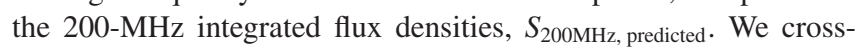
matched these sources with the results from the GLEAM sourcefinding, yielding 1178 matches within 2 arcmin positional threshold. From these, we excluded the sources with GLEAM $S_{200 \mathrm{MHz}}$, GLEAM $>1 \mathrm{Jy}$, leaving 43 sources with high signal-to-noise and clean SEDs. We compared the predicted flux densities to those measured by GLEAM in the wideband image, finding that $S_{200 \mathrm{MHz}}$, GLEAM $=$ $(0.80 \pm 0.13) S_{200 \mathrm{MHz} \text {, predicted }}$. We applied this factor to the final crossmatched ensemble of GLEAM integrated flux densities, yielding the 200-MHz measurements referred to as 'GLEAM' in Table 1.

We used the Positional Update and Matching Algorithm (PUMA, https://github.com/JLBLine/PUMA; Line et al. 2017) to establish the 

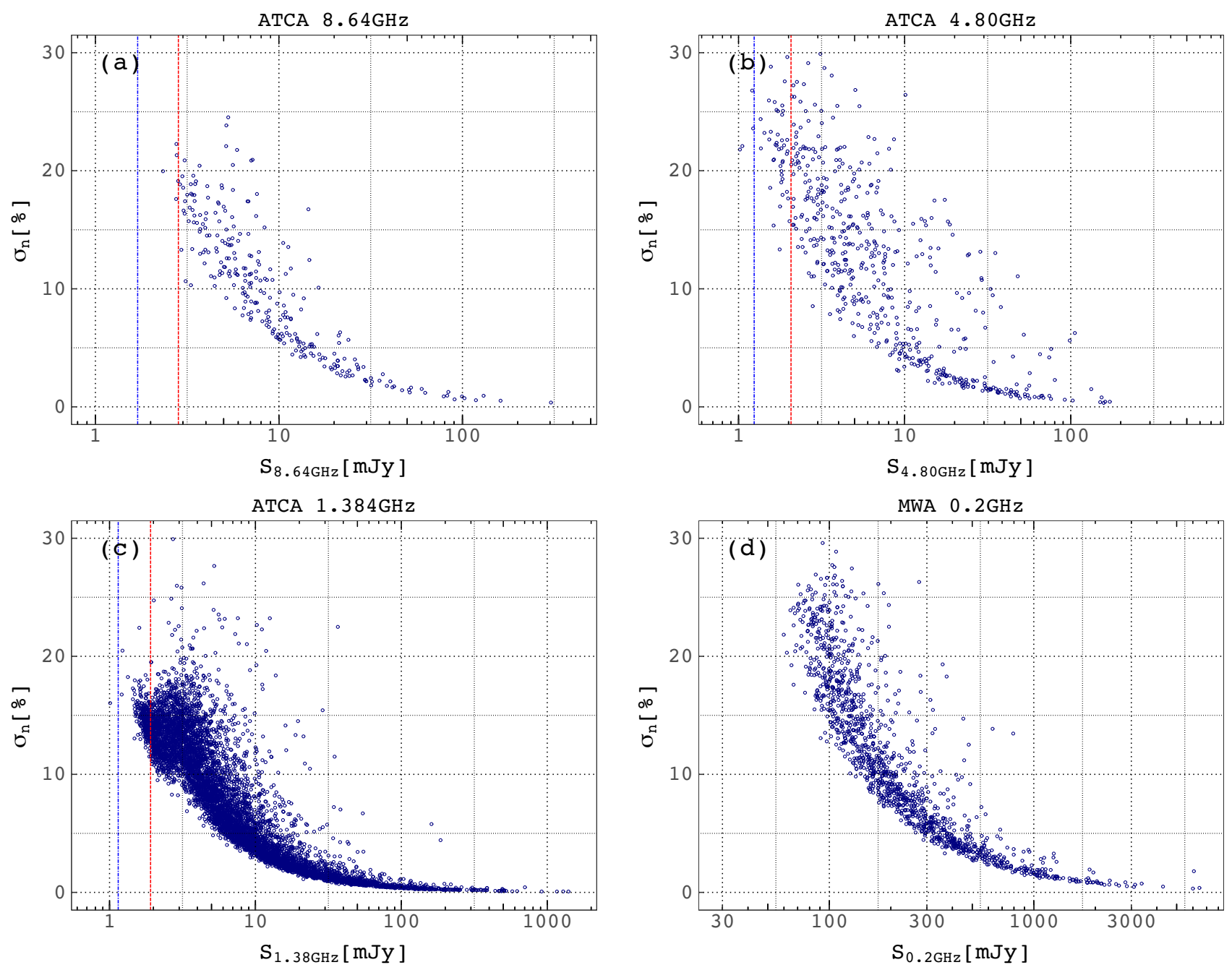

Figure 4. The fitting flux density error $\left(\sigma_{n}\right)$ for each source as a percent of the total integrated flux density. The blue dot-dashed line represents the $3 \sigma$ rms limit of each catalogue, and the $5 \sigma$ cut-off is shown with a red dotted line.

final cross-match between GLEAM and our main catalogue. PUMA is an open source software capable of cross-matching between radio surveys with different spectral (frequency) and resolving capabilities. The GLEAM catalogue was used as the base catalogue to match sources from our ATCA $1.384 \mathrm{GHz}$ catalogue within 2 arcmin and in combination with objects catalogued in the SUMSS $843 \mathrm{MHz}$ survey. Since PUMA relies on spectral characteristics of crossmatched sources, as well as on their positions, we used the newly re-calibrated GLEAM flux densities as described above. We adopted as positive matches all sources flagged by the PUMA algorithm as (a) isolated, i.e. only single cross-match is found within the search region, or (b) dominant, i.e. multiple cross-matches are found but one matched source fits a power law significantly better than the others (see the PUMA manual ${ }^{3}$ for more details). We found a set of 255 sources flagged as multiple, consisting of multiple higher resolution (see Section 3.5) sources blended into a single GLEAM source. For these objects, we have used GLEAM flux densities as upper limits for cross-matched, high resolution, components.

Finally, sources fainter than the source detection limit of $5 \sigma$ are considered undetected, and their flux density in our catalogue is

${ }^{3}$ https://github.com/JLBLine/PUMA given as an upper limit as $5 \sigma$, with the rescaling factor applied. We provide an additional column in the electronic catalogue with flag $(<)$ designating an upper limit for the flux density.

\subsection{Spectral index uncertainties}

Spectral index $(\alpha)$ is defined in Section 3.1. We use R's package METAFOR (Viechtbauer 2010) to estimate $\alpha$ using an unweighted mixed-effects linear regression model. The model takes into account uncertainties in the flux measurements to estimate $\alpha$ uncertainties. If no uncertainty is reported in the original measurement we assumed 10 percent of the flux value. Large uncertainties in spectral index $(\Delta \alpha>0.5)$ are mainly the result of only two frequencies available.

\section{RESULTS AND DISCUSSION}

\subsection{Optical and radio counterparts}

We make use of multiple optical catalogues (as described in Table 2) and we find 7883 unique optical sources that are thought to be sources behind the LMC. We apply a 5 arcsec search radius when matching optical counterparts to the highest frequency radio component. From 
Table 6. Example of the list (total of 343 sources) of sources from our base catalogue cross-matched with six optical surveys used in this study (see Section 2.6). We provide the name of the source as defined in Table 3, catalogued redshift value and the associated reference [columns $z$ and $z$ Ref. and the references to optical detection (Det. Ref.)]. Number-coded references are: (1) 6dF: Jones et al. (2009), (2) MACHO: Kim et al. (2012), MQS I: Kozłowski \& Kochanek (2009), (4) MQS II: Kozłowski et al. (2012), (5) MQS III: Kozłowski et al. (2013), and (6) MCELS: Smith \& MCELS Team (1998). The full catalogue is provided through the VIZIER service and as supplementary material.

\begin{tabular}{lccc}
\hline Name J & $z$ & $z$ Ref. & Det. ref. \\
\hline $041010-722358$ & 0.03201 & 1 & 1 \\
$041437-722726$ & 0.03207 & 1 & 1 \\
$041739-721901$ & 0.06528 & 1 & 1 \\
$042203-715315$ & $\ldots$ & $\ldots$ & 1 \\
$042529-735341$ & $\ldots$ & $\ldots$ & 1 \\
$\ldots$ & $\ldots$ & $\cdots$ & $\ldots$ \\
\hline
\end{tabular}

this, we find 343 unique optical sources (Table 6) of which 128 have spectroscopic redshifts. For these 128 sources, we calculate a mean redshift of 0.39 . The average positional offset between the radio continuum and optical sources is 1.59 and 1.72 arcsec in RA and Dec., respectively.

\subsection{AGN populations}

Kauffmann et al. (2003) used the Sloan Digital Sky Survey to argue that AGNs of all types and luminosities are typically found in the most massive galaxies. They also concluded that the optically luminous AGNs reside in galaxies with younger stellar populations with strong indications of recent starburst activity.

A subset of this population are the radio-loud AGNs that form highly collimated, fast jets of material that drive through the surrounding galaxy into the intergalactic medium, at close to the speed of light. This class of AGNs can be further divided into Quasars, BL Lacertae Objects (BL Lac) or Flat Spectrum Radio Quasars (FSRQ), Optically Violent Variable Quasars (OVV), and Radio Galaxies. Even though these jets are seen to primarily emit synchrotron radiation, their composition and production mechanism are still widely debated. These different classifications are thought to describe similar host galaxies (AGNs) seen at different orientation angles with respect to our line of sight, creating differences both in the observed radio morphology as well as the optical activity type (Antonucci 1993; Urry \& Padovani 1995; Urry 2003; Kazanas et al. 2012).

We use the definition from Sadler et al. (2006) to categorize the emission mechanisms of a source by its spectral index for centimetre wavelengths, i.e.: flat-spectrum sources with an $\alpha>-0.5$ are thought to be dominated by self absorbed, compact objects, while sources with $\alpha<-0.5$ are dominated by optically thin synchrotron emission (Collier et al. 2018; Galvin et al. 2018).

\subsubsection{GPS and CSS sources}

GPS and CSS sources are thought to contribute $~ 30-40$ per cent of radio sources (An \& Baan 2012; Lonsdale et al. 2016). However, their true nature is still under discussion. The debate about the absorption mechanism that contributes to the unique spectral features of this population has continued for over $30 \mathrm{yr}$. The most extensive study of GPS and CSS sources is presented by O'Dea (1998) and more recently O'Dea \& Saikia (2021) who hypothesized regarding their
Table 7. Example of the table that list properties of 123 IFRSs studied here. Column 2 is source IR name or IDs from SAGE and/or CatWISE2020 catalogue; Column 5 represents 3.4-3.6 $\mu \mathrm{m}$ flux density. SAGE is $3.6 \mu \mathrm{m}$ and CatWISE2020 $3.4 \mu \mathrm{m}$. The full catalogue is provided through the VIZIER service and as supplementary material.

\begin{tabular}{|c|c|c|c|c|c|}
\hline $\begin{array}{l}\text { ATCA Name } \\
\mathrm{J}\end{array}$ & $\begin{array}{l}\text { IR Source ID } \\
\text { CWISE }\end{array}$ & $\begin{array}{c}\mathrm{S}_{1.4 \mathrm{GHz}} \\
(\mathrm{mJy})\end{array}$ & $\begin{array}{c}\mathrm{S}_{843 \mathrm{MHz}} \\
(\mathrm{mJy})\end{array}$ & $\begin{array}{c}\mathrm{S}_{3.6 \mu \mathrm{m}} \\
(\mu \mathrm{Jy})\end{array}$ & $\alpha$ \\
\hline $041750-732120$ & J041750.62-732120.9 & 9.7 & 15.5 & 29.0 & -0.93 \\
\hline $041812-705127$ & J041812.62-705126.8 & 11.3 & 19.7 & 29.0 & -1.11 \\
\hline 041916-714548 & J041917.16-714548.1 & 6.8 & 11.7 & 8.6 & -1.07 \\
\hline 042034-704958 & J042034.04-704958.7 & 17.8 & 28.0 & 17.7 & -1.04 \\
\hline $042628-662829$ & J042628.58-662829.1 & 29.0 & 69.3 & 23.7 & -0.89 \\
\hline$\ldots$ & $\ldots$ & $\ldots$ & $\ldots$ & $\ldots$ & $\ldots$ \\
\hline
\end{tabular}

origin. They additionally use these sources as constraints on AGN physical evolution as it is believed that these sources represent the early stage of the AGN phase (Fanti et al. 1995; Polatidis \& Conway 2003; Fanti 2009; Randall et al. 2011; Callingham et al. 2017; Collier et al. 2018).

GPS and CSS sources are usually small (point) radio sources without jets and have similar characteristics in that they are both compact with steep spectra $(\alpha<-0.8)$. GPS sources have a much higher turnover frequency apart from their steep spectrum beyond the turnover frequency. This observable property allows their physical size to be estimated using the relation shown by Orienti \& Dallacasa (2014) who agree with previous work by O’Dea \& Baum (1997, equation 4). They define the relation (fit) as

$\log v_{m}=(-0.21 \pm 0.05)-(0.65 \pm 0.05) \log$ LLS,

where $v_{m}$ is the turnover frequency (in GHz) and LLS is the largest linear size (in kpc). This suggests a simple physical process common to both GPS and CSS populations that have been separately defined due to an arbitrary selection of turnover frequencies. Generally, it is believed that these objects are young, whose growth was frustrated by dense gas and dust or are simply young radio sources moving along their evolutionary path. Fanti et al. (1995) presented the 'young source' hypothesis, postulating that these sources typically have ages of $10^{6} \mathrm{yr}$ and tend to decrease their radio luminosity with time and grow in size from a few kpc to a few hundred kpc.

CSS sources are classified as such if they show an $\alpha$ of $<-0.8$, while GPS sources have an average $\alpha$ of +0.56 below the turnover frequency and -0.77 above the turnover. In this study, we detected 1866 CSS candidate sources with an $\alpha$ of $<-0.8$, indicating candidates for further analysis as discussed in Section 4.3.

\subsubsection{High Frequency Peakers}

We follow the convention given by Dallacasa et al. (2000) in naming radio continuum sources with spectra that peak above $5 \mathrm{GHz}$, High Frequency Peakers (HFPs). These high-frequency peaking sources are thought to be very young and compact, following the anticorrelation shown in equation (2). Additionally, these sources are thought to be rapidly changing in radio flux density, size, and flux density peak frequency on time-scales of a few decades. These sources are thought to then evolve into GPS sources, then become CSS sources and finally evolve into Large Scale Objects (LSOs) with a significantly broader (flatter; less peaked) spectrum (Fanti et al. 1995; Readhead et al. 1996; Alexander 2000; Marecki, Spencer \& Kunert 2003; Orienti \& Dallacasa 2008; An \& Baan 2012).

Dallacasa et al. (2000) presented a catalogue of 55 HFP sources above $300 \mathrm{mJy}$ near the peak frequency of $4.9 \mathrm{GHz}$, with the majority of these being unresolved sources at small angular scales. Additionally, they defined a sample of true HFPs as sources with an $\alpha$ 
Table 8. Median and mean spectral index distributions for each subset in the catalogue for sources that are detected at more than one radio frequency.

\begin{tabular}{lcccc}
\hline Subset & $\begin{array}{c}\text { Median } \\
\alpha\end{array}$ & $\begin{array}{c}\text { Mean } \\
\alpha\end{array}$ & $\begin{array}{c}\text { Standard } \\
\text { Deviation }\end{array}$ & $\begin{array}{c}\text { Number of } \\
\text { Sources }\end{array}$ \\
\hline All & -0.92 & -0.89 & 0.47 & 3789 \\
Resolution matched & -0.89 & -0.88 & 0.48 & 3636 \\
rms limited & -0.85 & -0.81 & 0.45 & 2984 \\
AT20G detection & -0.2 & -0.21 & 0.44 & 41 \\
No IR or optical & -0.95 & -0.93 & 0.46 & 2872 \\
IR counterpart & -0.84 & -0.78 & 0.47 & 917 \\
Optical counterpart & -0.71 & -0.66 & 0.49 & 209 \\
\hline
\end{tabular}

$<-0.56$ above the turnover frequency. Understanding and modelling these sources is of vital importance for three main reasons: (1) their evolutionary path is currently not very well understood; (2) these sources are highly variable on short time-scales, with this change likely driven by significant events in their host galaxy; (3) due to the high frequency of their emission peak, these sources can strongly contribute to measurements of the cosmic microwave background radiation as observed with the WMAP and PLANCK telescopes and as such these sources need to be accurately subtracted (De Zotti et al. 2000). Dallacasa \& Orienti (2016) show that a common behaviour of HFP sources is a spectral profile change (a change in time of the shape of the radio spectrum) correlated with an increase in optically thick emission and a decrease in optically thin emission.

We find 14 potential HFP characterized by an inverted spectrum (i.e. $\alpha>0.5$ ) and a possible turnover in their radio spectrum above $5 \mathrm{GHz}$, as discussed in Section 4.3.3.

\subsubsection{FSRQ and BL Lac candidates}

We investigated the sample of blazar candidates behind the LMC as presented by Żywucka et al. (2018). They identified 37 such objects (their tables 2 and 3 ) of which 23 are classified as FSRQ candidates and 14 are BL Lac candidates. Using a 30 arcsec search radius, we find 17 out of 23 FSRQ have a radio counterpart in common with the catalogue presented here (Table 3), and 9 out of 14 BL Lac candidates in common. We note that all 11 objects (6 FSRQ and 5 BL Lac candidates) that are not detected in our surveys are listed in Żywucka et al. (2018) as 'dubious objects'.

We denote these sources in our radio catalogue as the FSRQ or BL Lac accordingly (Table 3; Col. 13). The FSRQ candidate sample has a mean $\alpha$ of $-0.43 \pm 0.12$ (compared to Żywucka et al. 2018 findings of $-0.40 \pm 0.09$ ) while the BL Lac sample has a somewhat steeper mean $\alpha$ of $-0.72 \pm 0.13$, and a combined $\alpha$ of $-0.53 \pm 0.20$. This is expected to be flatter than the average AGN population $(\alpha=-0.89$; Table 8) as discussed in Section 4.3. We note that BL Lac objects are known to be highly variable and as such their spectral indices should be treated with caution as our radio continuum data were not obtained simultaneously. Additionally, we estimate the average optical redshift of FSRQ sample studied here is $1.37 \pm 0.17$ based on various $z$ measurements described in Section 2.6.

\subsubsection{IFRSs and $\mathrm{HzRG}$ candidates}

Finding High Redshift Radio Galaxies (HzRGs) is important in understanding the formation and evolution of galaxies at higher redshifts and in dense environments. Traditionally, radio sources with an Ultra Steep Spectrum (USS) $(\alpha<-1.3)$ have been the most efficient tracers of HzRGs at $z>2$. This technique is based on the observed steepening of the radio spectrum with both redshift and frequency. Almost all known HzRGs including the most distant one at $z=5.72$ (Saxena et al. 2018) have been identified using the USS selection method only. However, several studies (Waddington et al. 1999; Jarvis et al. 2009; Yamashita et al. 2020) demonstrated that the USS selection criterion is not fully efficient as they discovered radio galaxies with flatter spectral indices. Finding high redshift radioAGNs without relying on USS selection technique is now possible with the discovery of Infrared Faint Radio Sources (IFRSs) from the deep ATLAS radio continuum survey a decade ago.

IFRSs are a class of high redshift radio-loud AGNs originally identified as bright radio sources with no co-spatial IR counterpart when cross-matching the $1.4 \mathrm{GHz}$ ATLAS radio survey with the deep SWIRE IR survey between 3.6 and $24 \mu \mathrm{m}$ (Norris et al. 2006). As radio sources, the nature of IFRSs was unknown at the time. It was believed that all radio sources whether it is star-forming galaxies or AGNs would produce IR emission. Numerous follow-up studies have been conducted since their discovery to select more IFRSs and to understand what they are. Since the original selection criterion from Norris et al. (2006) was survey specific, Zinn, Middelberg \& Ibar (2011) redefined it and proposed a set of survey independent criteria, which even enable the selection of a brighter population of IFRSs with very faint IR counterpart. They have radio-to-IR flux density ratios above 500 and secondly, $3.6 \mu \mathrm{m}$ flux density less than $30 \mu \mathrm{Jy}$. To date, a total of $\sim 1400$ IFRSs have been identified from various radio surveys using the criteria of Zinn et al. (2011). All studies so far suggested that most of the known IFRSs, if not all, are radio-AGNs at $z>2$. Orenstein, Collier \& Norris (2019) further confirmed the high redshift nature of IFRSs by presenting the largest sample of 108 IFRSs with median spectroscopic redshift of 2.68. Additionally, some studies presented the evidence that IFRS population not only encompasses USS sources but also flat-spectrum sources too (Collier et al. 2014). Thus, selecting IFRSs serves as a valuable technique to discover all sorts of high redshift radio-AGNs.

We search for high redshift radio AGNs behind the LMC by selecting the potential IFRS candidates following the procedures from Collier et al. (2014) and Orenstein et al. (2019) utilizing the IR photometry from the CatWISE2020 (Marocco et al. 2021) and SAGE (Meixner et al. 2006) surveys. We cross-matched the radio sources in the LMC field with these two Mid-IR source catalogues, using a match radius of 1 arcsec. Because of the large radio beam sizes ( $\sim 40$ arcsec resolution), we chose 1 arcsec as the matching radius in order to minimize the false identifications. We acknowledge that by increasing the search radius we would certainly find many more true IFRS candidates but then the confidence level on these matches would be significantly lower.

A total of 123 IFRSs are identified in which 47 are found in SAGE, 71 in the CatWISE2020, and 5 in both. Out of 71 CatWISE2020 selected IFRSs 64 have a radio-to-IR flux density as defined by Zinn et al. (2011) larger than 500 at both $20 \mathrm{~cm}$ and $40 \mathrm{~cm}, 3$ at $20 \mathrm{~cm}$, and 11 at $40 \mathrm{~cm}$ only. Similarly, out of 47 SAGE-IFRSs 43 satisfied the Zinn ratio criteria at both $20 \mathrm{~cm}$ and $40 \mathrm{~cm}$, and the remaining 4 at $40 \mathrm{~cm}$ only. The five IFRSs identified in both SAGE and CatWISE2020, all have ratios above 500 at both $20 \mathrm{~cm}$ and $40 \mathrm{~cm}$. All of our IFRSs are found to have faint 3.4 or $3.6 \mu \mathrm{m}$ counterparts that are reliably detected (S/N > 5) in either of the CatWISE2020 or SAGE surveys. In Table $7,{ }^{4}$ we present our IFRSs sample. In Fig. 5, we present the spectral index distribution of our IFRSs sample, which follows a skewed distribution towards steep spectral indices with a median of -0.96 - very similar to the distribution shown by Collier et al. (2014).

${ }^{4}$ The full table will be in the supplementary material of the journal. 


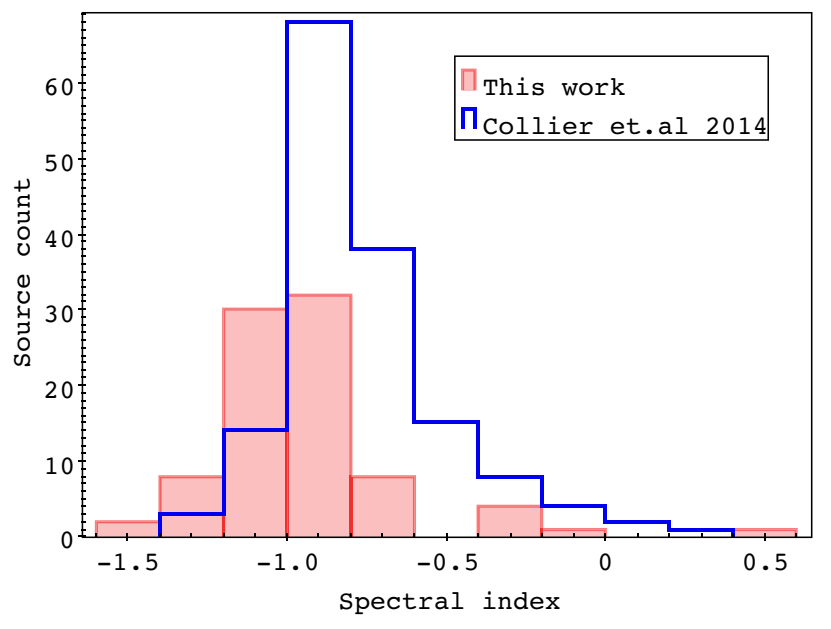

Figure 5. Radio spectral index distribution of our IFRSs sample in the field of the LMC, plotted as red solid boxes and IFRSs from Collier et al. (2014), plotted as blue dashed lines. Only sources with $\alpha_{N} \geq 3$ are plotted where $\alpha_{N}$ is the number of data points used to measure spectral index. Spectral index measurements in Collier et al. (2014) are based on 92, 20, and $6 \mathrm{~cm}$ data.

So far, only 131 IFRSs are known with spectroscopic redshifts lying in the range 1.63-4.4 (Collier et al. 2014; Orenstein et al. 2019). The only IFRSs in our sample with a measured redshift is J044537-685946 at $z=1.714$ (Żywucka et al. 2018).

To date, only two IFRSs have been reported with an X-ray counterpart, suggesting that they are AGNs of Type I (Collier et al. 2014). We analysed whether any of our IFRSs from this study had been detected in the XMM-Newton survey (Haberl 2019), and three IFRSs were found to have an X-ray counterpart within 15 arcsec radius. They are J051233-675037, J050634-675643, and J052350704213 , which we plan to follow-up for further studies.

\subsection{Spectral index for the entire sample}

CSS, GPS, Quasars, and distant AGNs cannot be distinguished easily by position in the sky in our catalogue as the angular resolutions of these existing radio surveys are, in most cases, insufficient. To remedy this, we have created a spectral index for each object to determine the dominant emission mechanism (see Table 3 ).

We perform a least-squares linear fit to model the source's spectral index through the use of a power-law consistent with the decay of synchrotron emission (a curved spectrum that would model synchrotron losses). However, fitting this simple single power-law model is not sufficient to categorize the turnover of GPS sources and other curved spectrum populations. Peacock (1985) showed that this type of analysis is only viable when applied to a small frequency range that ignores the effects of a curved spectrum.

As mentioned earlier, significant care must be taken when calculating the spectral index of a source. For instance, the resolution of each observation should be similar to avoid contributions from other sources. Initially, using all catalogues, we find a mean spectral index of $-0.89 \pm 0.47$ (and median of -0.92 ) from 3789 sources that are detected at two or more radio frequencies (Table 8). We subsequently refine this by presenting two spectral distributions; the first in Fig. 6 (left), utilizes all catalogues as presented in this paper. The second (and more accurate), Fig. 6 (right) uses only the catalogues at $0.843 \mathrm{GHz}$ and $1.384 \mathrm{GHz}$ given their similar resolution as shown in Table 1. Consequently, we find a median spectral index of -0.89 and mean of $-0.88 \pm 0.48$ from 3636 sources, summarized in Table 8 . However, one should bear in mind that these spectral indices are measured between two close frequency values $(0.843$ and $1.384 \mathrm{GHz}$ ) that are affected by larger uncertainties. Indeed, some 50 sources (less than 1 per cent of the total population) in Table 3 have questionable spectral index estimates of $\alpha<-2$. Where the spectral index values are extreme, we flag those sources to emphasis caution. The reason for such unrealistic spectral indices for these few sources is that the flux density measurements are made between
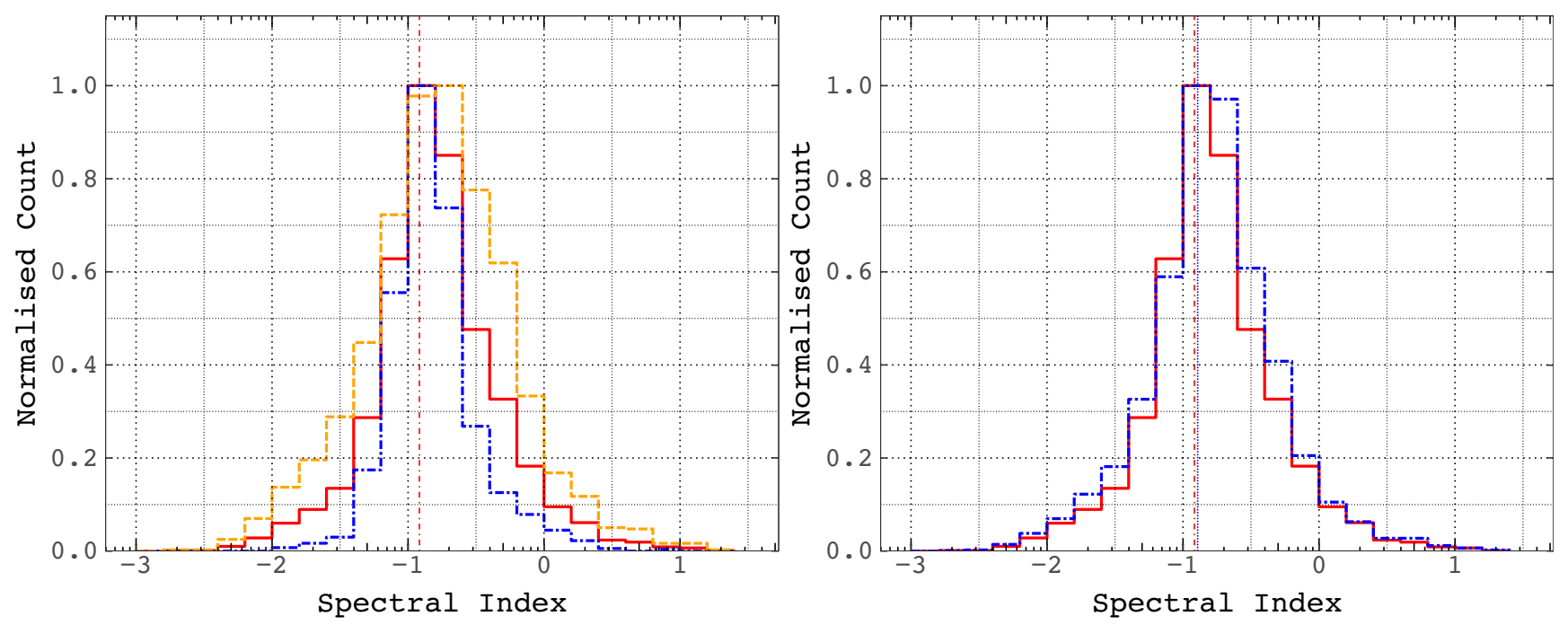

Figure 6. Left: Spectral index distribution for 3789 sources from all radio continuum catalogues. The red continuous line is the full catalogue, the orange dashed line is spectral indices estimated from sources with only two available data points, and blue dot-dashed line is estimates where more than two data points were available. The median $\alpha$ (vertical red dash-dotted line) for the full catalogue is -0.92 (Table 8). Right: A comparison between spectral index distributions for the full catalogue (red continuous line; same as in left figure) and for 3636 spectral indices estimated from two data points between $0.843 \mathrm{GHz}$ and $1.384 \mathrm{GHz}$ (blue dot-dashed line). The spectral index measure is restricted to these two frequencies as they provide similar angular resolutions. The median $\alpha$ for the $0.843 \mathrm{GHz}$ to $1.384 \mathrm{GHz}$ two-point spectral indices (vertical blue dotted line) is -0.89 (Table 8 ). Counts per bin are normalized to the maximum count per bin in each sample. 
Table 9. Source classification criteria for the radio continuum catalogue. $\dagger$ is unique sources detected at one or more frequencies.

\begin{tabular}{lccc}
\hline $\begin{array}{l}\text { Source } \\
\text { Classification }\end{array}$ & $\begin{array}{c}\text { Source } \\
\text { Count }\end{array}$ & $\begin{array}{c}\text { Classification } \\
\text { Criteria }\end{array}$ & $\begin{array}{c}\text { Catalogue } \\
\text { Flag }\end{array}$ \\
\hline All $\dagger$ & 6434 & & \\
Inverted $\alpha$ & 146 & $\alpha>0$ & USS \\
USS & 546 & $-1.3<\alpha<-0.8$ & CSS \\
CSS & 1866 & $100 \mathrm{MHz}<$ turnover $<5 \mathrm{GHz}$ & GPS \\
GPS & 10 & SED peak above $5 \mathrm{GHz}$ & HFP \\
HFP & 14 & various & IFRSs \\
IFRSs & 67 & & \\
\hline
\end{tabular}

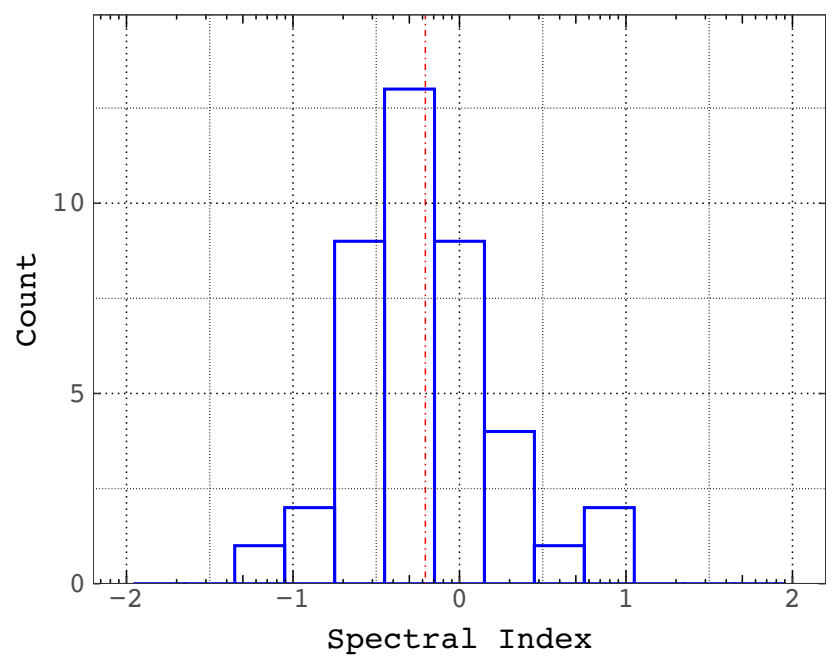

Figure 7. Spectral index distribution for 41 sources that have detection in the AT20G catalogue, measured using all radio continuum catalogues. The median $\alpha$ (vertical dash-dotted line) is -0.2 .

only two nearby frequency bands where a small change (or error) in size or flux density leads to large changes and somewhat unrealistic estimates in spectral index. Finally, looking at the median values of $\alpha$ in the last 3 lines of Table 8 , one can see that the more distant the sources are (or the fewer optical/IR counterparts they have) the steeper their spectral index.

From this analysis, we find $1866(\sim 25$ percent $)$ sources with an $\alpha<-0.8$ that we classify as CSS source candidates. Additionally, we find 0 sources that have a highly inverted spectrum $(\alpha>0.5)$ that we classify as potential HFP sources in which the turnover is unconstrained due to our limited frequency range, as shown in Table 9.

We closely inspected the 41 AT20G sources in our catalogue (as shown in Fig. 7), and found that the spectral index distribution of these sources is significantly different from the distribution for the catalogue as a whole (shown in Fig. 6). This distribution has a mean $\alpha$ of $-0.21 \pm 0.44$ (Table 8 ). Among these 41 sources, we find 1 CSS, 0 GPS, 14 HFP, and 0 Potential High Frequency Peaker sources that heavily skew the overall spectral index distribution towards $\alpha \sim$ 0 . This is due to a selection bias as we are selecting only sources with a detection in a shallower high frequency survey that tends to select bright sources with flat or inverted spectra. However, in practice we are biased towards FSRQ or BL Lacs.

Interestingly, sources that have an optical counterpart tend to have a flatter spectral index as shown in Fig. 8 and summarized in Table 8. For example, we find the mean spectral index for this subsample to

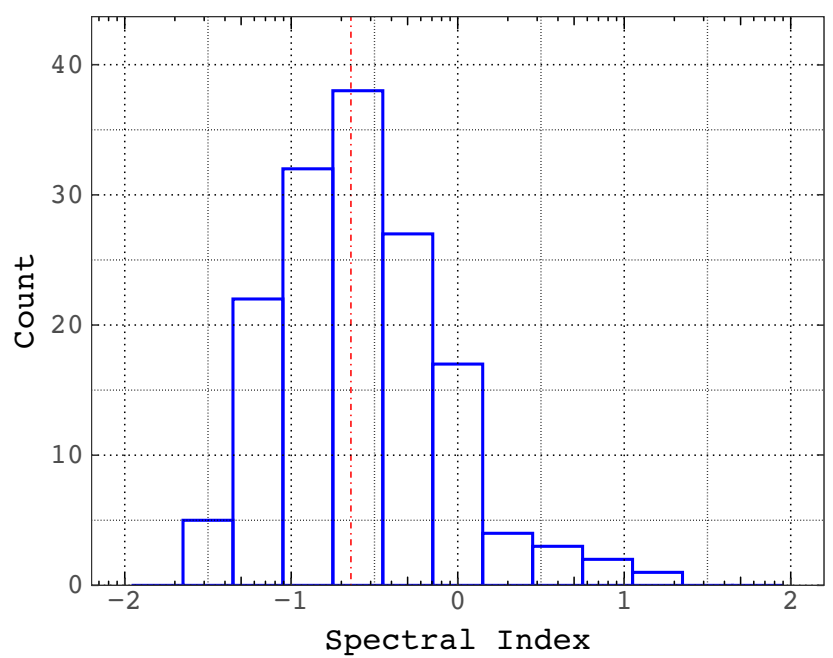

Figure 8. Spectral index distribution for 209 sources that have a detection in at least one optical catalogue, measured using all radio continuum catalogues. The median $\alpha$ (vertical dash-dotted line) is -0.71 .

be $-0.66 \pm 0.49$ from 209 sources (Table 8). This is also reflected in radio sources that have an IR counterpart, but without an optical detection. Specifically, these sources have a mean spectral index of $-0.78 \pm 0.47$ from 917 sources. In contrast, sources that do not have an IR or optical counterpart, have a far steeper mean spectral index at $-0.93 \pm 0.46$ from 2872 sources (Table 8 ). As for the AT20G sample, this is probably a selection bias. These optical counterparts are mainly QSOs or BL Lacs with well-established flat spectral index. Similarly, but to a lesser extent, IR detected sources are more likely to have a strong IR AGN component, typical of a QSO and/or Seyfert 1 AGN, that tends to have flatter radio spectra. This interpretation seems supported by the fact that sources with no IR counterpart have steeper radio spectra on average, i.e. are more dominated by radio galaxies.

\subsubsection{Sensitivity limitations}

Almost all sources (6434 sources, 100 percent) in our main catalogue (Table 3) are detected at $0.843 \mathrm{GHz}$ or $1.384 \mathrm{GHz}$. The remaining 0 sources are weak sources detected in either the 4.8 or $8.64 \mathrm{GHz}$ catalogues. These 0 sources may represent either variable sources or HFP (peaking their flux density above $5 \mathrm{GHz}$ ) as discussed in Section 4.3.3.

For a $5 \sigma$ source to be included in the $1.384 \mathrm{GHz}$ catalogue, an integrated flux density of at least $2.5 \mathrm{mJy}$ is required (at the average rms noise of $0.5 \mathrm{mJy}$ beam $^{-1}$ ). A $2.5 \mathrm{mJy}$ source in the ATCA $1.384 \mathrm{GHz}$ catalogue is expected to appear in the SUMSS catalogue only if it has a $0.843 \mathrm{GHz}$ flux of $6 \mathrm{mJy}$ ( 5 times the average rms), which would imply a very extreme spectral index of -1.7 . For a more standard spectral index of -0.7 only sources with $1.384 \mathrm{GHz}$ flux densities larger than 3.5 mJy can have a counterpart in the SUMSS catalogue.

This clearly limits the study of the spectral index of the $1.384 \mathrm{GHz}$ source catalogue at the low flux density end, since $5-10 \sigma 1.384 \mathrm{GHz}$ sources might not be detected at $843 \mathrm{GHz}$ due to the higher flux density limit of the SUMSS catalogue. It also introduces a bias in the study of the spectral index of the faintest $1.384 \mathrm{GHz}$ sources, as only very steep sources can be detected at $0.843 \mathrm{GHz}$. This bias against flat-spectrum sources at the low $(3-10 \sigma) 1.384 \mathrm{GHz}$ flux densities can be seen in Fig. 9 (right-hand panel), where we show the 

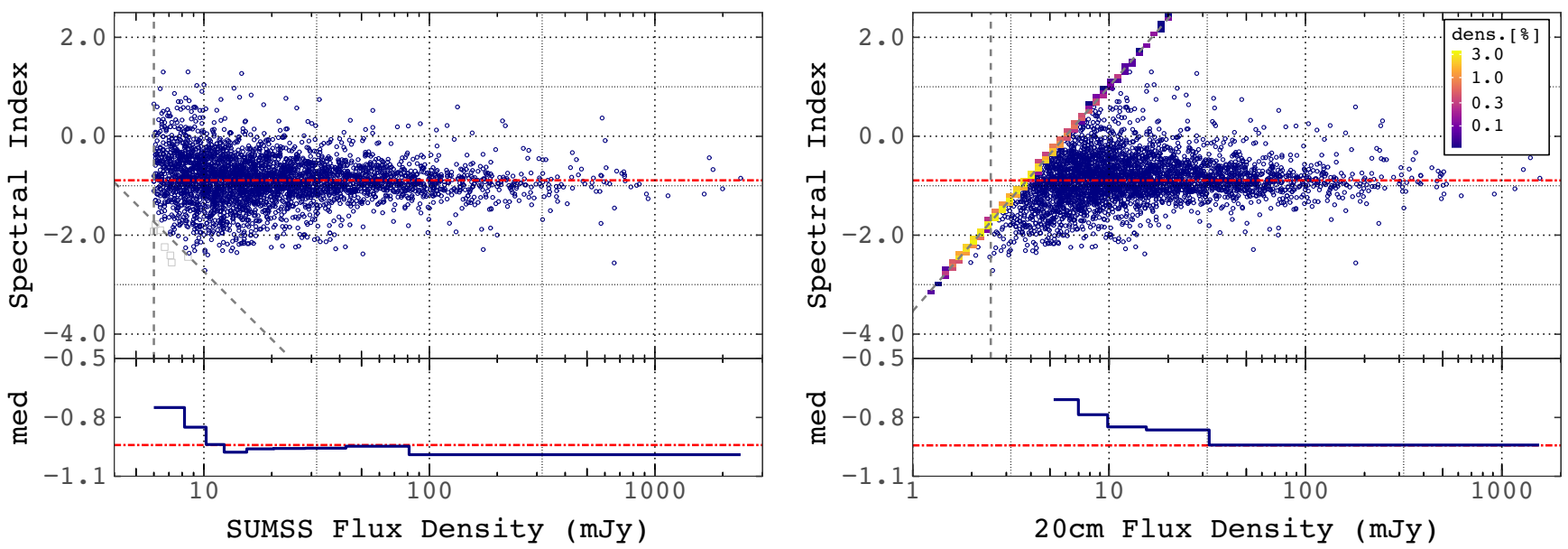

Figure 9. Integrated flux density versus spectral index for the two most complete survey lists: SUMSS (left) and ATCA at 1.384 GHz (top panels) and the distribution of spectral index medians per flux density bin (bottom panels). Vertical dashed lines in top panels indicate flux limits for each survey. Red horizontal dot-dashed lines in all panels represent the median spectral index $(-0.89)$ for the complete sample of all sources in common to SUMSS and ATCA surveys. For the ATCA $1.384 \mathrm{GHz}$ data points with no counterparts at $0.843 \mathrm{GHz}$ we show the approximate source counts (in per cent) of the limiting spectral indices as a binned density map (scale bar shown at the top right corner). For the median versus flux density plots data is split in nine equally populated bins. Note that the bins with more points from limiting than calculated spectral indices are not shown.

Table 10. Spectral index statistics for the $1.384 \mathrm{GHz}$ selected catalogue (ATCA), as derived in different flux density bins.

\begin{tabular}{lccccc}
\hline $\begin{array}{l}\text { Lower } \\
(\mathrm{mJy})\end{array}$ & $\begin{array}{c}\text { Upper } \\
(\mathrm{mJy})\end{array}$ & $\mathrm{N}$ & $\begin{array}{c}\text { Median } \\
(\alpha)\end{array}$ & $\begin{array}{c}\text { Mean } \\
(\alpha)\end{array}$ & $\sigma_{\alpha}$ \\
\hline 4.69 & 5.96 & 642 & -0.51 & -0.71 & 0.53 \\
5.96 & 7.94 & 642 & -0.67 & -0.64 & 0.61 \\
7.94 & 10.96 & 643 & -0.77 & -0.65 & 0.64 \\
10.96 & 17.38 & 642 & -0.81 & -0.69 & 0.67 \\
17.38 & 35.48 & 642 & -0.83 & -0.73 & 0.59 \\
35.48 & 1548.82 & 643 & -0.89 & -0.79 & 0.75 \\
\hline
\end{tabular}

Table 11. Spectral index statistics for the $0.843-\mathrm{GHz}$ selected catalogue (SUMSS), as derived in different flux density bins.

\begin{tabular}{lccccc}
\hline $\begin{array}{l}\text { Lower } \\
(\mathrm{mJy})\end{array}$ & $\begin{array}{c}\text { Upper } \\
(\mathrm{mJy})\end{array}$ & $\mathrm{N}$ & $\begin{array}{c}\text { Median } \\
(\alpha)\end{array}$ & $\begin{array}{c}\text { Mean } \\
(\alpha)\end{array}$ & $\sigma_{\alpha}$ \\
\hline 6.00 & 8.00 & 373 & -.68 & -0.67 & 0.57 \\
8.00 & 9.79 & 369 & -0.79 & -0.79 & 0.61 \\
9.79 & 11.75 & 359 & -0.89 & -0.90 & 0.53 \\
11.75 & 14.45 & 362 & -0.89 & -0.94 & 0.53 \\
14.45 & 17.78 & 357 & -0.95 & -0.97 & 0.49 \\
17.78 & 22.91 & 360 & -0.89 & -0.89 & 0.43 \\
22.91 & 31.62 & 363 & -0.92 & -0.92 & 0.42 \\
31.62 & 47.86 & 361 & -0.91 & -0.92 & 0.38 \\
47.86 & 89.13 & 361 & -0.90 & -0.87 & 0.31 \\
89.13 & 2398.83 & 363 & -0.95 & -0.95 & 0.35 \\
\hline
\end{tabular}

spectral index between $0.843 \mathrm{GHz}$ and $1.384 \mathrm{GHz}$ as a function of the integrated flux densities for the ATCA $1.384 \mathrm{GHz}$ sources. The diagonal dashed line shows the limit of the region of the spectral index - flux parameter space where we can detect the source at both frequencies due to the limited sensitivity of the $0.843 \mathrm{GHz}$ SUMSS catalogue. For sources above this line only spectral index limits can be derived. A similar bias can be in principle introduced when studying the spectral index of the SUMSS sources, this time against steep spectrum sources. Such a bias can, however, be neglected in our case, as the $1.384 \mathrm{GHz}$ ATCA catalogue is more sensitive, and the faintest SUMSS sources (i.e. those detected at $5 \sigma=6 \mathrm{mJy}$ ) can all have a counterpart in the $1.384 \mathrm{GHz}$ ATCA catalogue, except for very extreme (and rare) cases where the spectral index is steeper than -1.7. Indeed, Fig. 9 (left-hand panel) shows that only a few SUMSS sources are not detected at $1.384 \mathrm{GHz}$ (upper limits are shown as open grey squares at the bottom left).

\subsubsection{Spectral flattening}

Spectral flattening at low frequencies $(\leq 5 \mathrm{GHz})$ has been suggested in the literature, from studies of deep radio surveys. It is conjectured that the faint radio source populations of a few mJy have a flatter mean spectral index than brighter source populations. For instance, Prandoni et al. (2006) studied the spectral index distribution of a sub-sample of sources extracted from the 1.4 and $5 \mathrm{GHz}$ Australia Telescope ESO Slice Project surveys, finding a flatter mean (1.4$5 \mathrm{GHz}$ ) spectral index of $-0.53 \pm 0.05$ for sources with $\mathrm{S}_{1.4 \mathrm{GHz}} \leq$ $4 \mathrm{mJy}$, when compared to $-0.66 \pm 0.05$ for sources above $4 \mathrm{mJy}$. These authors observed a similar behaviour for the $5 \mathrm{GHz}$ selected sample, with mean spectral indices of $-0.24 \pm 0.06$ and $-0.58 \pm 0.06$, respectively, below and above $4 \mathrm{mJy}$, in line with previous results based on smaller samples (Donnelly, Partridge \& Windhorst 1987; Fomalont et al. 1991). The flatter values at $5 \mathrm{GHz}$ were expected as higher frequency surveys tend to select source populations with flatter spectra. More recently, Owen et al. (2009) pushed the spectral index analysis to lower flux densities $\left(\mathrm{S}_{1.4 \mathrm{GHz}}>10 \mu \mathrm{Jy}\right)$ and lower frequencies $(0.330 \mathrm{MHz})$, finding similar results: in the SWIRE deep field they measured median $(0.33-1.4 \mathrm{GHz})$ spectral indices of $-0.54 \pm 0.06$ in the flux interval $\mathrm{S}_{1.4 \mathrm{GHz}} \sim 0.3-1 \mathrm{mJy}$, and $0.66 \pm 0.11$ at larger flux densities. Both Prandoni et al. (2006) and Owen et al. (2009) found that this flattening is mostly associated with compact sources, likely core dominated AGN. Interestingly, Owen et al. (2009) find a significant re-steepening at $\mathrm{S}_{1.4 \mathrm{GHz}}<0.3 \mathrm{mJy}$, which is interpreted as the result of star-forming galaxies becoming the dominant population in the $\mu \mathrm{Jy}$ regime.

It should be noted, however, that the tendency of radio source spectra to become flatter with decreasing flux density is not observed by all authors. For instance, Randall et al. (2012) and Zinn et al. 

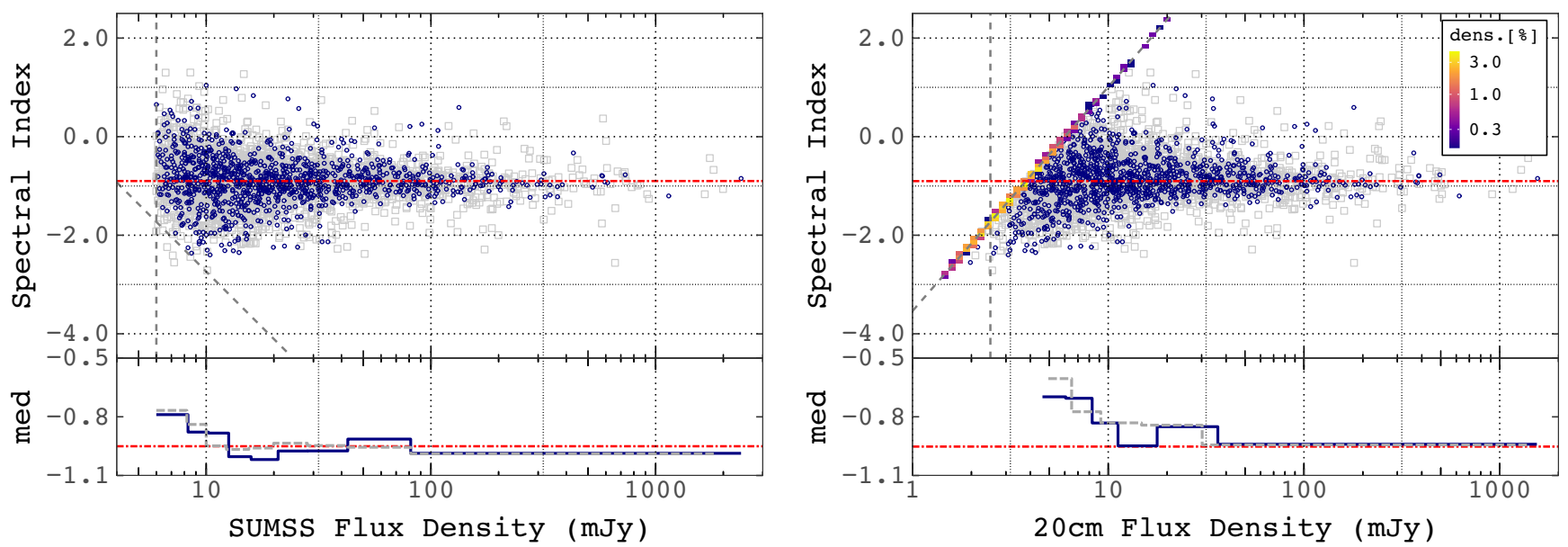

Figure 10. As in Fig. 9 but splitting the sample into two groups: Open circles represent sources within $3.5^{\circ}$ from the LMC centre (inner subsample) while open squares are sources outside this radius (outer subsample). In the bottom panels, we show the distribution of spectral index medians per, equally populated, flux density bin for the inner subsample (blue solid line) and outer subsample (grey dashed line) separately. No significant difference in source spectral index distribution is observed between the two groups, indicating that our catalogue is mainly comprised of background sources.

(2012) found no statistical spectral flattening for mJy sources. This, however, could be a consequence of not well-matched sensitivities at the various frequencies, which might prevent the spectral index analysis to be applied to the faintest sources, as discussed in Section 4.3.1. Indeed, the $1.4 \mathrm{GHz}$ survey by Zinn et al. (2012) has an rms $\sim 3$ times lower than that of their $2.3 \mathrm{GHz}$ survey.

In order to provide further clues on this matter, we searched for spectral index - flux density trends in our LMC field catalogue. Tables 10 and 11 show the mean and median values of the spectral index as a function of 1.384 and $0.843 \mathrm{GHz}$ flux densities, respectively. The median values are also shown in the bottom panels of Fig. 9. As discussed in Section 4.3.1, the limited sensitivity of the $0.843 \mathrm{GHz}$ catalogue makes it unreliable for any spectral index analysis of the faintest $1.384 \mathrm{GHz}$ selected sources, as only very steep-spectrum sources can be detected at both frequencies and spectral index limits become dominant. For this reason, we decided to restrict our analysis of the $1.384 \mathrm{GHz}$ ATCA sample to flux densities where spectral index limits represent less than 50 per cent of the total number of sources in the flux bin under consideration, allowing us to get reliable median spectral index values. This happens at $\mathrm{S}_{1.4 \mathrm{GHz}}>4.7 \mathrm{mJy}$. For the shallower SUMSS catalogue, we do not have significant biases and the analysis can be pushed down to the catalogue flux limit (i.e. $6 \mathrm{mJy}$ ).

We notice that the spectral flattening reported in previous works is observed at lower flux densities than those probed by this study. Nevertheless, the larger statistics available allows us to split our sources in finer flux density bins. This in turn allows us to identify a small but clear trend towards flatter spectral indices already at $\mathrm{S}<10 \mathrm{mJy}$. We also notice that median spectral index values tend to be flatter for sources in the $1.384 \mathrm{GHz}$ catalogue than for those in the $0.843 \mathrm{GHz}$ catalogue, consistent with the results of Prandoni et al. (2006), where flatter spectral index values were found for the higher frequency selected sample.

Focusing on the $1.384 \mathrm{GHz}$ catalogue, where spectral index values can be directly examined, we find that a median ${ }^{5}$ spectral index value of -0.51 at $S \sim 5 \mathrm{mJy}$ is consistent with the -0.53 found by Prandoni et al. (2006) at $\mathrm{S}<4 \mathrm{mJy}$ and the -0.54 found by Owen et al.

${ }^{5}$ The mean spectral index value in this flux bin is not well constrained due to the many spectral index limits.
(2009) at $\mathrm{S}<1 \mathrm{mJy}$, indicating we may be probing the same radio source population. Also, consistent with the mean values measured at higher fluxes: they vary from -0.64 to -0.69 in the flux range 6-17 mJy, against a value of -0.66 reported by Prandoni et al. (2006) at $\mathrm{S}>4 \mathrm{mJy}$.

In order to test if there is any difference in spectral index distribution (including a possible contribution to the source spectral flattening) of sources belonging to the LMC, we constructed the same graphs for the sources inside of the $3.5^{\circ}$ radius from the approximate centroid of the LMC at RA $=79.5 \mathrm{deg}$ and Dec. $=-68.9 \mathrm{deg}$ (Fig. 10). We found no difference in source spectral index distribution within or outside the $3.5^{\circ}$ radius (filled circles and open squares, respectively), indicating that our catalogue is mainly comprised of background sources.

\subsubsection{Radio two-colour diagram}

We adopt the radio 'two-colour' diagram as shown by Sadler et al. (2006) in order to allow the identification of sources with curved spectra (Fig. 11). This approach treats the high and low frequency components of each source separately, by calculating two spectral indices. Here, we define our high frequency sample as $>1.5 \mathrm{GHz}$ and our low frequency sample as the inverse case (i.e. $<1.5 \mathrm{GHz}$ ). This analysis should be treated with caution as the spectral index was calculated based on flux densities from observations with different angular resolutions.

Using the radio two-colour diagram, we find 10 sources with a spectral index of $\alpha_{1.5}^{20 \mathrm{GHz}} \leq 0 \leq \alpha_{0.2}^{1.5 \mathrm{GHz}}$. This limited sample is primarily due to the lower source density at $20 \mathrm{GHz}$.

We used a second order polynomial to determine the turnover frequency for GPS candidates. In Fig. 12, we show an example of radio spectra of one of the objects from this sample fitted with second order polynomial.

With these estimated turnover frequencies, we use equation (2) to determine the LLS for each GPS candidate, as shown in Table 12. The average turnover frequency for this sample of GPS sources is $1.73 \mathrm{GHz}$ with a corresponding average LLS of $320 \mathrm{pc}$. These results are consistent with findings of Jeyakumar (2016).

We find 14 sources that have a convex spectrum with a peaked (turnover) integrated flux density above $5 \mathrm{GHz}$ and, as such, we 

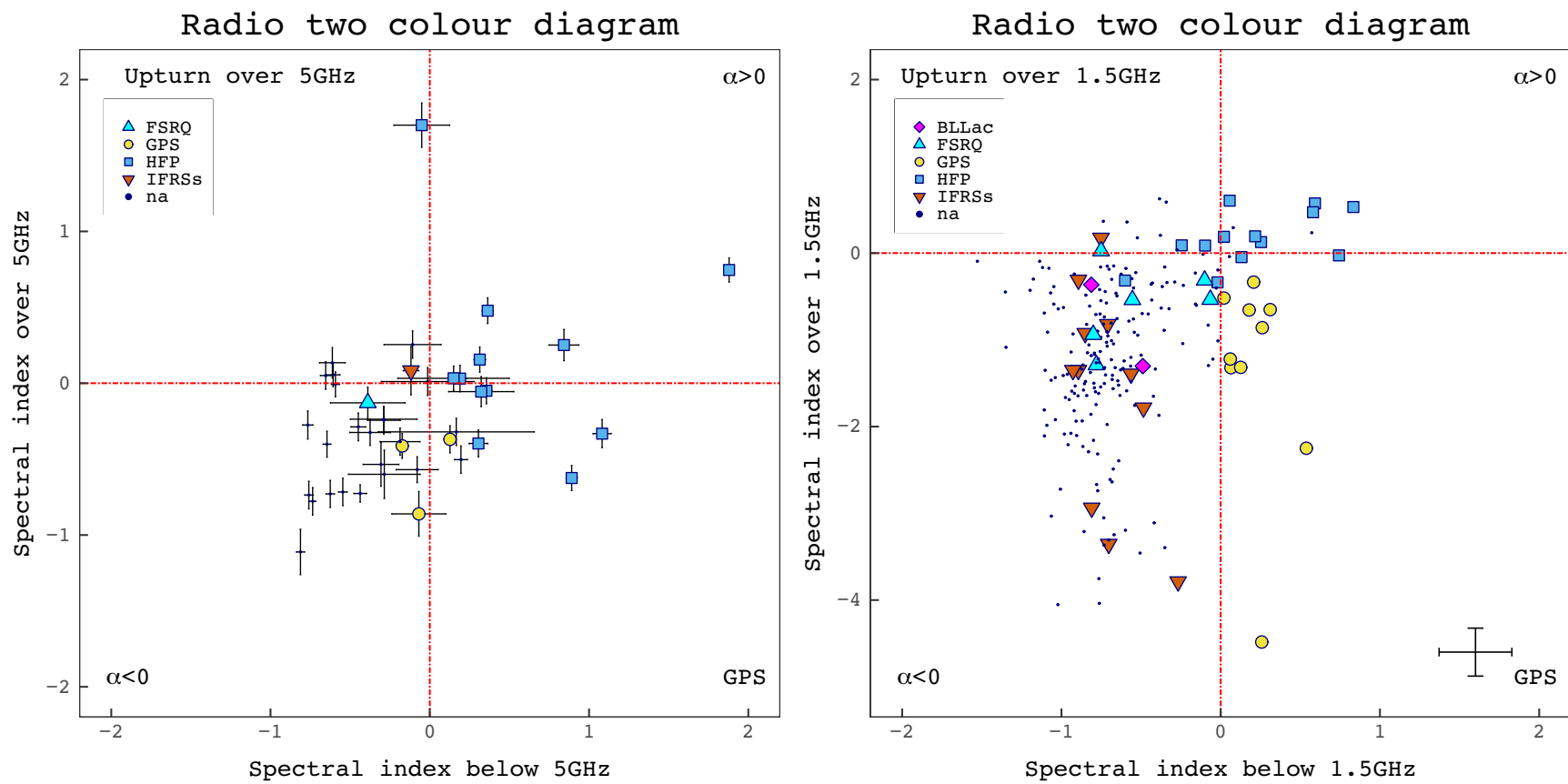

Figure 11. $5 \mathrm{GHz}$ radio 'two-colour' diagram (left) for 41 sources, contrasting two spectral index measurements for each source divided at $5 \mathrm{GHz}$. The median $\alpha$ for the low frequency measurement is -0.17 and the mean $\alpha$ for the high frequency measurement is $-0.29 .1 .5 \mathrm{GHz}$ radio two-colour diagram (right) for 234 sources, contrasting two spectral index measurements for each source divided at $1.5 \mathrm{GHz}$. The median $\alpha$ for the low frequency measurement is -0.71 and the mean $\alpha$ for the high frequency measurement is -1.04 . In the lower right corner, we show median errors in estimated spectral indices. In the upper left corner (both plots), we show the colour code of the points for different classes presented here.

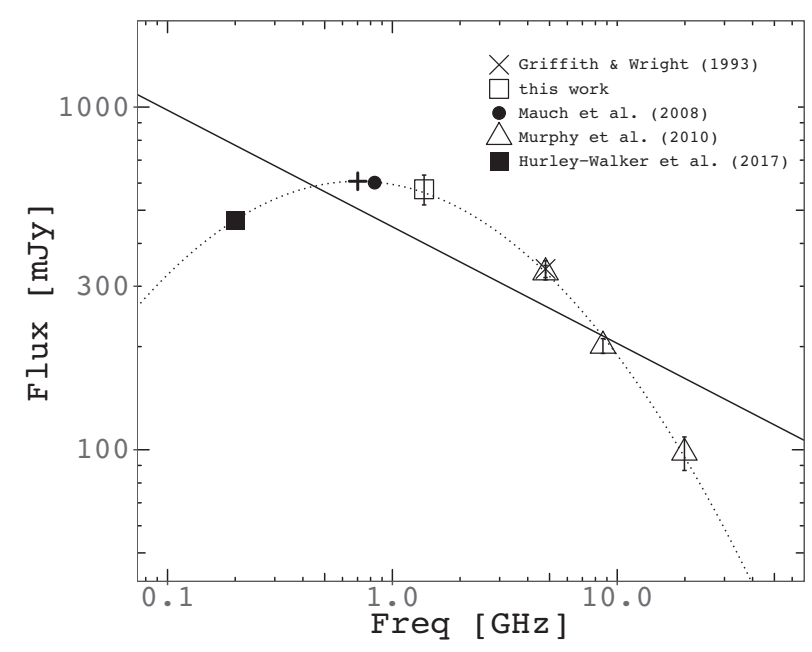

Figure 12. Example of the radio spectra of one of the GPS candidates (J061011-724814) fitted with a second order polynomial (dotted line) and power-law function (continuous line). A cross $(+)$ represents the location of the estimated turnover frequency.

follow Dallacasa et al. (2000) classification and label them as HFP sources.

\section{SUMMARY AND CONCLUSION}

We have compiled a master catalogue based on several new and existing radio continuum lists of sources behind the LMC, to a $5 \sigma$ detection level from $\sim 200 \mathrm{mJy}$ down to $0.2 \mathrm{mJy}$, depending on observing frequency (see Table 1). From this, a total of 6434 sources
Table 12. Turnover frequency and linear size relation (using equation 2) for our sample of GPS candidate sources.

\begin{tabular}{lccc}
\hline Source name & $\begin{array}{c}\text { Turnover } v \\
(\mathrm{GHz})\end{array}$ & $\begin{array}{c}\text { LLS } \\
(\mathrm{kpc})\end{array}$ & $\begin{array}{c}\text { Candidate } \\
\text { Type }\end{array}$ \\
\hline $045358-713817$ & 2.05 & 0.16 & GPS \\
$045516-705446$ & 2.71 & 0.10 & GPS \\
$050000-632237$ & 1.00 & 0.47 & GPS \\
$050139-662525$ & 1.05 & 0.44 & GPS \\
$052310-720624$ & 1.76 & 0.20 & GPS \\
$052505-664052$ & 2.29 & 0.13 & GPS \\
$052517-672246$ & 1.62 & 0.23 & GPS \\
$054317-662655$ & 3.14 & 0.08 & GPS \\
$054736-680905$ & 0.94 & 0.52 & GPS \\
$061011-724813$ & 0.70 & 0.82 & GPS \\
\hline
\end{tabular}

have been identified, with multifrequency detections of 3789 sources. We find the median spectral index of $\alpha=-0.89$ for 3636 sources which are detected at two frequencies $(0.843$ and $1.384 \mathrm{GHz})$ with similar resolution (FWHM $\sim 40-45$ arcsec). We have cross-matched these 6434 sources with and five optical surveys (see Table 2). As a result, we find optical counterparts for 343, of which 128 have a redshift measurement with a mean redshift of 0.39 .

Based on the SUMSS and $1.384 \mathrm{GHz}$ ATCA source lists, we present evidence that radio sources below $10 \mathrm{mJy}$ exhibit flatter radio spectra than brighter ones. To take into account the biases introduced by the limited sensitivity of the $0.843 \mathrm{GHz}$ SUMSS catalogue, we restricted our analysis to $1.384 \mathrm{GHz}$ flux densities $\mathrm{S}>5 \mathrm{mJy}$. As a result, we obtain median $(0.843-1.384 \mathrm{GHz})$ spectral indices flattening from values of $<-0.8$ for $S_{1.4 \mathrm{GHz}}>10 \mathrm{mJy}$ to 0.51 for $\mathrm{S}_{1.4 \mathrm{GHz}}<5 \mathrm{mJy}$. This is in general agreement with findings from previous deep surveys (Prandoni et al. 2006; Owen et al. 2009). 
Additionally, 1866 unresolved sources have been detected with a spectral index of $<-0.8$ between $408 \mathrm{MHz}$ and $20 \mathrm{GHz}$ ( $\mathrm{Sec}-$ tion 4.2.1). This is a strong indicator for young AGNs that are either CSS or GPS objects. Furthermore, we find evidence for 10 of these sources to be GPS candidates, which have $\alpha_{5 \mathrm{GHz}}^{20 \mathrm{GHz}} \leq 0 \leq \alpha_{843 \mathrm{MHz}}^{5 \mathrm{GHz}}$. We classify 14 sources as HFP and 0 as possible HFP sources (see Table 9) that have steep inverted spectra where we do not observe a turnover (i.e. the turnover frequency is $>20 \mathrm{GHz}$ ).

\section{ACKNOWLEDGEMENTS}

The Australian SKA Pathfinder is part of the Australia Telescope National Facility that is managed by Australian Commonwealth Scientific and Industrial Research Organisation (CSIRO). Operation of ASKAP is funded by the Australian Government with support from the National Collaborative Research Infrastructure Strategy. ASKAP uses the resources of the Pawsey Supercomputing Centre. Establishment of ASKAP, the Murchison Radio-astronomy Observatory and the Pawsey Supercomputing Centre are initiatives of the Australian Government, with support from the Government of Western Australia and the Science and Industry Endowment Fund. We acknowledge the Wajarri Yamatji people as the traditional owners of the Observatory site. This work was supported by resources provided by the Pawsey Supercomputing Centre with funding from the Australian Government and the Government of Western Australia. This paper includes archived data obtained through the Australia Telescope Online Archive (http://atoa.atnf.csiro.au). The Australia Telescope Compact Array is part of the Australia Telescope National Facility, which is funded by the Australian Government for operation as a National Facility managed by CSIRO. Parts of this research were conducted with the support of Australian Research Council Centre of Excellence for All Sky Astrophysics in 3 Dimensions (ASTRO 3D), through project number CE170100013. IP acknowledges support from INAF under the SKA/CTA PRIN 'FORECaST' and the PRIN MAIN STREAM 'SAuROS' projects. HA has benefited from grant CIIC 174/2021 of Universidad de Guanajuato, Mexico. DU acknowledges Ministry of Education, Science and Technological Development of the Republic of Serbia through contract No. 445103-9/2021-14/200104.

\section{DATA AVAILABILITY}

The catalogues underlying this article are available on the CDS/VIZIER (https://cds.u-strasbg.fr/) website. The full source list (Tables 4, 5, 6, and 7) is also provided through the CDS/VIZIER service.

\section{REFERENCES}

Alexander P., 2000, MNRAS, 319, 8

An T., Baan W. A., 2012, ApJ, 760, 77

Antonucci R., 1993, ARA\&A, 31, 473

Bock D. C.-J., Large M. I., Sadler E. M., 1999, AJ, 117, 1578

Bozzetto L. M. et al., 2017, ApJS, 230, 2

Callingham J. R. et al., 2017, ApJ, 836, 174

Collier J. D. et al., 2014, MNRAS, 439, 545

Collier J. D. et al., 2018, MNRAS, 477, 578

Condon J. J., Cotton W. D., Greisen E. W., Yin Q. F., Perley R. A., Taylor G. B., Broderick J. J., 1998, AJ, 115, 1693

Dallacasa D., Orienti M., 2016, Astron. Nachr., 337, 120

Dallacasa D., Stanghellini C., Centonza M., Fanti R., 2000, A\&A, 363, 887

De Zotti G., Granato G. L., Silva L., Maino D., Danese L., 2000, A\&A, 354, 467
Dickel H. R., 2000, Balt. Astron., 9, 564

Dickel J. R., McIntyre V. J., Gruendl R. A., Milne D. K., 2005, AJ, 129, 790

Donnelly R. H., Partridge R. B., Windhorst R. A., 1987, ApJ, 321, 94

Fanti C., 2009, Astron. Nachr., 330, 120

Fanti C., Fanti R., Dallacasa D., Schilizzi R. T., Spencer R. E., Stanghellini C., 1995, A\&A, 302, 317

Filipovic M. D., Haynes R. F., White G. L., Jones P. A., Klein U., Wielebinski R., 1995, A\&AS, 111, 311

Filipovic M. D., White G. L., Jones P. A., Haynes R. F., Pietsch W. N., Wielebinski R., Klein U., 1996a, in Burkert A., Hartmann D. H., Majewski S. A., eds, ASP Conf. Ser. Vol. 112, The History of the Milky Way and Its Satellite System. Astron. Soc. Pac., San Francisco, p. 91

Filipovic M. D., White G. L., Haynes R. F., Jones P. A., Meinert D., Wielebinski R., Klein U., 1996b, A\&AS, 120, 77

Filipovic M. D., Jones P. A., White G. L., Haynes R. F., 1998a, A\&AS, 130, 441

Filipovic M. D., Haynes R. F., White G. L., Jones P. A., 1998b, A\&AS, 130, 421

Filipovic M. D., Jones P. A., White G. L., Haynes R. F., 1998c, Publ. Astron. Soc. Aust., 15, 128

Filipović M. D. et al., 2009, MNRAS, 399, 769

Fomalont E. B., Windhorst R. A., Kristian J. A., Kellerman K. I., 1991, AJ, 102,1258

For B.-Q. et al., 2018, MNRAS, 480, 2743

Gaensler B. M., Haverkorn M., Staveley-Smith L., Dickey J. M., McClureGriffiths N. M., Dickel J. R., Wolleben M., 2005, Science, 307, 1610

Galvin T. J. et al., 2018, MNRAS, 474, 779

Gregory P. C., Condon J. J., 1991, ApJS, 75, 1011

Griffith M. R., Wright A. E., 1993, AJ, 105, 1666

Haberl F., 2019, in A Synoptic View of the Magellanic Clouds: VMC, Gaia and Beyond, p. 63

Hancock P. J., Murphy T., Gaensler B. M., Hopkins A., Curran J. R., 2012a, Astrophysics Source Code Library, record ascl:1212.009

Hancock P. J., Murphy T., Gaensler B. M., Hopkins A., Curran J. R., 2012b, MNRAS, 422, 1812

Hancock P. J., Trott C. M., Hurley-Walker N., 2018, Publ. Astron. Soc. Aust., $35, \mathrm{e} 011$

Harvey-Smith L., Hardwick J. A., De Marco O., Parthasarathy M., Gonidakis I., Akhter S., Cunningham M., Green J. A., 2018, MNRAS, 479, 1842

Haynes R. F. et al., 1991, A\&A, 252, 475

Heywood I. et al., 2016, MNRAS, 457, 4160

Hilditch R. W., Howarth I. D., Harries T. J., 2005, MNRAS, 357, 304

Hopkins A. M. et al., 2015, Publ. Astron. Soc. Aust., 32, e037

Hotan A. W. et al., 2014, Publ. Astron. Soc. Aust., 31, e041

Hughes A., Wong T., Ekers R., Staveley-Smith L., Filipovic M., Maddison S., Fukui Y., Mizuno N., 2006, MNRAS, 370, 363

Hughes A., Staveley-Smith L., Kim S., Wolleben M., Filipović M., 2007, MNRAS, 382, 543

Hurley-Walker N. et al., 2017, MNRAS, 464, 1146

Jarrett T. H., Chester T., Cutri R., Schneider S., Skrutskie M., Huchra J. P., 2000, AJ, 119, 2498

Jarvis M. J., Teimourian H., Simpson C., Smith D. J., Rawlings S., Bonfield D., 2009, MNRAS, 398, L83

Jeyakumar S., 2016, MNRAS, 458, 3786

Jones D. H., Saunders W., Read M., Colless M., 2005a, Publ. Astron. Soc. Aust., 22, 277

Jones H., Saunders W., Colless M., Read M., Parker Q., Watson F., Campbell L., 2005b, in Fairall A. P., Woudt P. A., eds, ASP Conf. Ser. Vol. 329 , Nearby Large-Scale Structures and the Zone of Avoidance. Astron. Soc. Pac., San Francisco, p. 11

Jones D. H. et al., 2009, MNRAS, 399, 683

Joye W. A., Mandel E., 2003, in Payne H. E., Jedrzejewski R. I., Hook R. N., eds, ASP Conf. Ser. Vol. 295, Astronomical Data Analysis Software and Systems XII. Astron. Soc. Pac., San Francisco, p. 489

Kaczmarek J. F., Purcell C. R., Gaensler B. M., McClure-Griffiths N. M., Stevens J., 2017, MNRAS, 467, 1776

Kauffmann G. et al., 2003, MNRAS, 346, 1055 
Kazanas D., Fukumura K., Behar E., Contopoulos I., Shrader C., 2012, Astron. Rev., 7, 92

Kim D.-W., Protopapas P., Trichas M., Rowan-Robinson M., Khardon R., Alcock C., Byun Y.-I., 2012, ApJ, 747, 107

Kozłowski S., Kochanek C. S., 2009, ApJ, 701, 508

Kozłowski S., Kochanek C. S., Udalski A., 2011, ApJS, 194, 22

Kozłowski S. et al., 2012, ApJ, 746, 27

Kozłowski S. et al., 2013, ApJ, 775, 92

Large M. I., Mills B. Y., Little A. G., Crawford D. F., Sutton J. M., 1981, MNRAS, 194, 693

Leahy D., Wang Y., Lawton B., Ranasinghe S., Filipović M., 2019, AJ, 158, 149

Leverenz H., Filipović M. D., Vukotić B., Urošević D., Grieve K., 2017, MNRAS, 468, 1794

Line J. L. B., Webster R. L., Pindor B., Mitchell D. A., Trott C. M., 2017, Publ. Astron. Soc. Aust., 34, e003

Lonsdale C. J. et al., 2016, Astron. Nachr., 337, 194

Maggi P. et al., 2016, A\&A, 585, A162

Maggi P. et al., 2019, A\&A, 631, A127

Mao S. A. et al., 2012, ApJ, 759, 25

Marecki A., Spencer R. E., Kunert M., 2003, Publ. Astron. Soc. Aust., 20, 46

Marocco F. et al., 2021, ApJS, 253, 8

Mathewson D. S., Cleary M. N., Murray J. D., 1974, ApJ, 190, 291

Mauch T., Murphy T., Buttery H. J., Curran J., Hunstead R. W., Piestrzynski B., Robertson J. G., Sadler E. M., 2003, MNRAS, 342, 1117

Meixner M. et al., 2006, AJ, 132, 2268

Middelberg E. et al., 2008, AJ, 135, 1276

Mills B. Y., Turtle A. J., 1984, in van den Bergh S., de Boer K. S. D., eds, Proc. IAU Symp. 108, Structure and Evolution of the Magellanic Clouds. Springer, Netherland, p. 283

Murphy T. et al., 2010, MNRAS, 402, 2403

Norris R. P. et al., 2006, AJ, 132, 2409

O’Dea C. P., 1998, PASP, 110, 493

O’Dea C. P., Baum S. A., 1997, AJ, 113, 148

O’Dea C. P., Saikia D. J., 2021, A\&AR, 29, 3

Ochsenbein F., Bauer P., Marcout J., 2000, A\&AS, 143, 23

Orenstein B. J., Collier J. D., Norris R. P., 2019, MNRAS, 484, 1021

Orienti M., Dallacasa D., 2008, A\&A, 477, 807

Orienti M., Dallacasa D., 2014, MNRAS, 438, 463

Owen F. N., Morrison G. E., Klimek M. D., Greisen E. W., 2009, AJ, 137, 4846

Parker Q. A., Bojičić I. S., Frew D. J., 2016, J. Phys.: Conf. Ser., 728, 032008

Payne J. L., Filipovic M. D., Millar W. C., Crawford E. J., de Horta A. Y., Stootman F. H., Urosevic D., 2008, Serb. Astron. J., 177, 53

Payne J. L., Tauber L. A., Filipovic M. D., Crawford E. J., de Horta A. Y., 2009, Serb. Astron. J., 178, 65

Peacock J. A., 1985, MNRAS, 217, 601

Pennock C. M. et al., 2021, MNRAS, 506, 3540

Pietrzyński G. et al., 2013, Nature, 495, 76

Pietrzyński G. et al., 2019, Nature, 567, 200

Polatidis A. G., Conway J. E., 2003, Publ. Astron. Soc. Aust., 20, 69

Prandoni I., Parma P., Wieringa M. H., de Ruiter H. R., Gregorini L., Mignano A., Vettolani G., Ekers R. D., 2006, A\&A, 457, 517

Randall K. E., Hopkins A. M., Norris R. P., Edwards P. G., 2011, MNRAS, 416, 1135

Randall K. E., Hopkins A. M., Norris R. P., Zinn P.-C., Middelberg E., Mao M. Y., Sharp R. G., 2012, MNRAS, 421, 1644

Readhead A. C. S., Taylor G. B., Pearson T. J., Wilkinson P. N., 1996, ApJ, 460, 634

Reid W. A., 2014, MNRAS, 438, 2642

Reid W. A., Parker Q. A., 2013, MNRAS, 436, 604

Reynolds J., 1994, Atnf Technical Memos, 39.3 040, http://www.atnf.csiro .au/observers/memos/d96783 1.pdf

Riess A. G., Casertano S., Yuan W., Macri L. M., Scolnic D., 2019, ApJ, 876, 85

Sadler E. M. et al., 2006, MNRAS, 371, 898

Saxena A. et al., 2018, MNRAS, 480, 2733
Skrutskie M. F. et al., 2006, AJ, 131, 1163

Smith R. C., MCELS Team, 1998, Publ. Astron. Soc. Aust., 15, 163

Taylor M. B., 2005, in Shopbell P., Britton M., Ebert R., eds, ASP Conf. Ser. Vol. 347, Astronomical Data Analysis Software and Systems XIV. Astron. Soc. Pac., San Francisco, p. 29

Taylor M., 2011, Astrophysics Source Code Library, record ascl:1101.010

Udalski A. et al., 1994, Acta Astron., 44, 165

Urry M., 2003, in Collin S., Combes F., Shlosman I., eds, ASP Conf. Ser. Vol. 290, Active Galactic Nuclei: From Central Engine to Host Galaxy. Astron. Soc. Pac., San Francisco, p. 3

Urry C. M., Padovani P., 1995, PASP, 107, 803

Viechtbauer W., 2010, J. Stat. Softw., 36, 1

Vukotić B., Urošević D., Filipović M. D., Payne J. L., 2009, A\&A, 503, 855

Waddington I., Windhorst R. A., Cohen S. H., Partridge R. B., Spinrad H., Stern D., 1999, ApJ, 526, L77

Wayth R. B. et al., 2015, Publ. Astron. Soc. Aust., 32, e025

Wenger M. et al., 2000, A\&AS, 143, 9

Wright E. L. et al., 2010, AJ, 140, 1868

Yamashita T. et al., 2020, AJ, 160, 60

Yew M. et al., 2021, MNRAS, 500, 2336

Zinn P. C., Middelberg E., Ibar E., 2011, A\&A, 531, A14

Zinn P.-C., Middelberg E., Norris R. P., Hales C. A., Mao M. Y., Randall K. E., 2012, A\&A, 544, A38

Żywucka N., Goyal A., Jamrozy M., Stawarz Ł., Ostrowski M., Kozłowski S., Udalski A., 2018, ApJ, 867, 131

\section{SUPPORTING INFORMATION}

Supplementary data are available at MNRAS online.

Table 3. Example of the point source catalogue of 6434 objects in the directions of the LMC with its positions, integrated flux densities with associated uncertainty and spectral index.

Table 4. Example for the source list (total of 1995 sources) at $0.843 \mathrm{GHz}$ derived from ASKAP-Beta survey.

Table 5. Example for the source list (total of 2084 sources) at $0.843 \mathrm{GHz}$ derived from MOST.

Table 6. Example of the list (total of 343 sources) of sources from our base catalogue cross-matched with six optical surveys used in this study (see Section 2.6).

Table 7. Example of the table that list properties of 123 IFRSs studied here. Column 2 is source IR name or IDs from SAGE and/or CatWISE2020 catalogue; Column 5 represents 3.4-3.6 $\mu \mathrm{m}$ flux density.

Please note: Oxford University Press is not responsible for the content or functionality of any supporting materials supplied by the authors. Any queries (other than missing material) should be directed to the corresponding author for the article.

${ }^{1}$ School of Science, Western Sydney University, Locked Bag 1797, Penrith South DC, NSW 2751, Australia

${ }^{2}$ CSIRO Astronomy and Space Science, PO Box 76, Epping, NSW 1710, Australia

${ }^{3}$ Minnesota Institute for Astrophysics, School of Physics and Astronomy, University of Minnesota, 116 Church Street SE, Minneapolis, MN 55455, USA

${ }^{4}$ INAF - Istituto di Radioastronomia, Via P. Gobetti 101, I-40129 Bologna, Italy

${ }^{5}$ Depto. de Astronomía, DCNE, Universidad de Guanajuato, Cjón. de Jalisco s/n, Col. Valenciana, Guanajuato, CP 36023, Gto., Mexico

${ }^{6}$ International Centre for Radio Astronomy Research, Curtin University, Bentley, WA 6102, Australia

${ }^{7}$ The Inter-University Institute for Data Intensive Astronomy (IDIA), Department of Astronomy, University of Cape Town, Rondebosch 7701, South Africa 
${ }^{8}$ ARC Centre of Excellence for All Sky Astrophysics in 3 Dimensions (ASTRO 3D), Australia

${ }^{9}$ International Centre for Radio Astronomy Research, University of Western Australia, 35 Stirling Hwy, Crawley, WA 6009, Australia

${ }^{10}$ Max-Planck-Institut für extraterrestrische Physik, Gießenbachstraße 1, D85748 Garching, Germany

${ }^{11}$ Australian Astronomical Optics, AAO-Macquarie, Faculty of Science and Engineering, Macquarie University, 105 Delhi Rd, North Ryde, NSW 2113 Australia

${ }^{12}$ INAF - Osservatorio Astrofisico di Catania, via Santa Sofia 78, I-95123 Catania, Italia

${ }^{13}$ School of Cosmic Physics, Dublin Institute for Advanced Studies, 31 Fitzwilliam Place, Dublin 2, Ireland

${ }^{14}$ Dominion Radio Astrophysical Observatory, Herzberg Astronomy and Astrophysics, National Research Council Canada, PO Box 248, Penticton, BC V2A 6J9, Canada

${ }^{15}$ Department of Physics and Astronomy, University of Calgary, Calgary, $A B$ T2N 1N4, Canada

${ }^{16}$ Observatoire Astronomique de Strasbourg, Université de Strasbourg, CNRS, 11 rue de l'Université, F-67000 Strasbourg, France
${ }^{17}$ National Radio Astronomy Observatory, 1003 Lopezville Road, Socorro, NM 87801, USA

${ }^{18}$ Department of Physics, Earth Science and Space Systems Engineering, Morehead State University, 235 Martindale Drive, Morehead, KY 40351, USA

${ }^{19}$ Lennard-Jones Laboratories, Keele University, Staffordshire ST5 5BG, UK

${ }^{20}$ School of Physical Sciences, The University of Adelaide, Adelaide 5005, Australia

${ }^{21}$ National Astronomical Observatory of Japan, Mitaka, Tokyo 181-8588, Japan

${ }^{22}$ Remeis Observatory and ECAP, Universität Erlangen-Nürnberg, Sternwartstraße 7, D-96049 Bamberg, Germany

${ }^{23}$ Department of Astronomy, Faculty of Mathematics, University of Belgrade, Studentski trg 16, 11000 Belgrade, Serbia

${ }^{24}$ Isaac Newton Institute of Chile, Yugoslavia Branch

${ }^{25}$ Thüringer Landessternwarte, Sternwarte 5, D-07778 Tautenburg, Germany

This paper has been typeset from a $\mathrm{T}_{\mathrm{E}} \mathrm{X} / \mathrm{L} \mathrm{T}_{\mathrm{E}} \mathrm{X}$ file prepared by the author. 